\title{
Addition of Improved Shock-Capturing Schemes to OVERFLOW 2.1
}

\author{
Robert W. Tramel ${ }^{1}$ \\ Kord Technologies Inc., Huntsville, Alabama 35804 \\ Robert H. Nichols ${ }^{2}$ \\ University of Alabama Birmingham, Birmingham, Alabama 35294 \\ and \\ Pieter G. Buning ${ }^{3}$ \\ NASA Langley Research Center, Hampton, Virginia, 23681
}

Existing approximate Riemann solvers do not perform well when the grid is not aligned with strong shocks in the flow field. Three new approximate Riemann algorithms are investigated to improve solution accuracy and stability in the vicinity of strong shocks. The new algorithms are compared to the existing upwind algorithms in OVERFLOW 2.1. The new algorithms use a multidimensional pressure gradient based switch to transition to a more numerically dissipative algorithm in the vicinity of strong shocks. One new algorithm also attempts to artificially thicken captured shocks in order to alleviate the errors in the solution introduced by "stair-stepping" of the shock resulting from the approximate Riemann solver. This algorithm performed well for all the example cases and produced results that were almost insensitive to the alignment of the grid and the shock.

\section{Nomenclature}

\begin{tabular}{|c|c|}
\hline$a$ & $=$ speed of sound \\
\hline$\hat{a}$ & $=$ Roe averaged speed of sound \\
\hline$e_{t}$ & $=$ total energy per unit mass \\
\hline$\vec{F}$ & $=$ inviscid flux vector \\
\hline$k_{p}$ & $=$ pressure switch sensor \\
\hline$k_{T}$ & $=$ temperature switch sensor \\
\hline$p$ & $=$ pressure \\
\hline$S$ & $=$ wave speed \\
\hline$t$ & $=$ time \\
\hline$\vec{U}$ & $=$ conserved variable vector \\
\hline$u$ & $=$ velocity in the $\mathrm{x}$ direction \\
\hline $\bar{u}$ & $=$ contact discontinuity speed \\
\hline$\hat{u}$ & $=$ Roe averaged velocity in the $\mathrm{x}$ direction \\
\hline$x$ & $=$ spatial direction \\
\hline$\beta$ & $=$ switching function \\
\hline$\hat{\lambda}$ & $=$ Roe averaged eigenvalue of the inviscid flux jacobians \\
\hline$\rho$ & $=$ density \\
\hline$\xi, \eta, \zeta$ & $=$ computational coordinates \\
\hline
\end{tabular}

${ }^{1}$ Chief Scientist, AIAA Senior Member.

${ }^{2}$ Research Associate Professor, Department of Mechanical Engineering, AIAA Senior Member.

${ }^{3}$ Aerospace Engineer, Computational Aerosciences Branch, Mail Stop 128, AIAA Associate Fellow. 


\section{Subscripts}

$$
\begin{array}{ll}
i & =\text { spatial index } \\
L & =\text { left state } \\
\mathrm{M} & =\text { contact discontinuity } \\
R & =\text { right state }
\end{array}
$$

\section{Superscripts}

$$
\begin{array}{ll}
n & =\text { temporal index } \\
* & =\text { intermediate state }
\end{array}
$$

\section{Introduction}

$\mathrm{T}_{\mathrm{i}}^{\mathrm{h}}$ he accurate computation of supersonic and hypersonic flows place conflicting demands on the formulation of inviscid numerical flux functions. A high speed numerical scheme must possess sufficient dissipation to capture strong shocks without developing overshoots and oscillations in the vicinity of the discontinuity. The scheme must also possess numerical dissipation that is much smaller than the physical viscosity to accurately compute boundary layers. The simultaneous satisfaction of these two requirements makes the computation of viscous hypersonic flows extremely challenging, particularly at the higher Mach numbers where many of the best schemes experience numerical difficulties.

Most numerical algorithms developed for high speed flows are based on an approximate solution of the Riemann problem. Approximate Riemann solvers such as those developed by Roe ${ }^{1}$ or Harten, Lax, and van Leer (HLL) ${ }^{2}$ are widely used for high speed flows because of their numerical accuracy and robustness. These approximate solvers are derived for one dimensional flow, and extensions to two and three dimensions introduce a possible source for numerical errors. Approximate Riemann schemes are highly sensitive to how well the shock is aligned with the grid. For cases in which the grid is not aligned with the shock, cross coupling between the Riemann problems in the different directions occurs. The strong jump in the conserved variables across the shock is "interpreted" by the Riemann solvers in the non-aligned directions as strong jumps in the other characteristic variables. This introduces numerical error for schemes that possess numerical dissipation that scales directly with the cell interface normal velocity in directions tangential to the shock where this component of velocity is low. The lack of dissipation in these tangential directions causes the cross-coupling perturbations to be either amplified (for schemes based on the use of anti-diffusion) or to be neutrally stable, i.e., the disturbances propagate without damping. In both cases the solution quality is severely degraded, resulting in non-physical features often referred to as "carbuncles".

Ad hoc fixes ${ }^{2,3,4,5}$ to the eigenvalues based on satisfying entropy conditions have been proposed as a remedy to the difficulties with non-grid aligned shocks in the past, but all such approaches are problematic in satisfying the conflicting requirements for hypersonic shock capturing. Some of the fixes severely degrade the solution in the boundary layer. In addition, these entropy corrections merely attempt to add dissipation rather than directly manipulating the anti-diffusion terms. It is reasonable to expect that this effect would be especially aggravated in the case of tetrahedral grids ${ }^{6}$ where the cell interfaces would likely be badly misaligned with the shock discontinuity. In this effort three new flux formulations are implemented into the OVERFLOW 2.1 flow solver and their ability to accurately compute flows with strong shocks and their efficacy is demonstrated on a variety of test cases.

\section{Theory}

The Godunov family of numerical schemes may be examined using the 1-D Euler equations

$$
\frac{\partial \vec{U}}{\partial t}+\frac{\partial \vec{F}}{\partial x}=0
$$

Here $\vec{U}$ is the vector of conserved variables $\left[\rho, \rho u, \rho e_{t}\right]^{\mathrm{T}}$ and $\vec{F}$ is the inviscid flux vector in the direction given by 


$$
\vec{F}=\left[\begin{array}{c}
\rho \\
\rho u^{2}+p \\
\left(\rho e_{t}+p\right) u
\end{array}\right]
$$

Eq. (1) can be discretized to obtain

$$
\vec{U}_{i}^{n+1}=\vec{U}_{i}^{n}-\frac{\Delta t}{\Delta x}\left(\vec{F}_{i+1 / 2}-\vec{F}_{i-1 / 2}\right)
$$

where $\Delta t$ and $\Delta x$ are temporal and spatial increments respectively.

\section{Roe Scheme}

The Roe $^{1}$ approximate Riemann solver defines the interface numerical flux as

$$
\vec{F}_{R o e}=\frac{1}{2}\left(\vec{F}_{L}+\vec{F}_{R}\right)-\frac{1}{2}|\vec{A}|\left(\vec{U}_{R}-\vec{U}_{L}\right)
$$

where $\vec{A}$ is the Roe averaged inviscid flux Jacobian matrix given by

$$
\vec{A}=\hat{L} \hat{\lambda} \hat{R}
$$

$\hat{\lambda}_{1,2,3}=[\hat{u}, \hat{u}+\hat{a}, \hat{u}-\hat{a}]$ are the Roe-averaged eigenvalues of the inviscid flux Jacobians and $\hat{L}$ and $\hat{R}$ are the left and right Roe-averaged eigenvectors of the inviscid fluxes. The Roe scheme has a well known deficiency in enforcing the entropy condition (i.e. in preventing nonphysical expansion shocks) when the eigenvalues of $\vec{A}$ vanish in regions near sonic points or stagnation points. This deficiency has traditionally been addressed by limiting the eigenvalues such that they do not fall below an arbitrary minimum value. The entropy fix implemented in OVERFLOW ${ }^{7}$ uses the following function to select the eigenvalues used in the flux reconstruction.

$$
\lambda_{i}=\operatorname{MAX}\left\{\left[\frac{\left|\lambda_{R_{i}}\right|}{\lambda_{R_{i}}}\left(\lambda_{R_{i}}-\lambda_{L_{i}}\right)-\frac{\left|\lambda_{L_{i}}\right|}{\lambda_{L_{i}}}\left(\lambda_{R_{i}}-\lambda_{L_{i}}\right), j,\left|\hat{\lambda}_{i}\right|_{\mid}\right\}\right.
$$

\section{HLLEM Scheme}

Harten, Lax and Van Leer ${ }^{2}$ proposed a family of Godunov schemes know as the HLL approximate Riemann solvers. The HLL formulation simplifies the solution to the Riemann problem at the cell interfaces by using averaged values for the interior states. The simplest HLL scheme assumes only one interior state between the fastest left and right running waves $\left(\mathrm{S}_{\mathrm{L}}\right.$ and $\left.\mathrm{S}_{\mathrm{R}}\right)$. These waves represent the smallest and largest signal speeds with which information of the initial discontinuity is transported. A schematic ( $x-t$ plot) of the HLL formulation is depicted in Fig. 1. For Einfeldt's version ${ }^{8}$ of the HLL scheme (denoted as HLLE), the single state Riemann solution is given by

$$
\vec{U}_{H L L}=\left\{\begin{array}{c}
\vec{U}_{L} \text { if } S_{L}>0 \\
\vec{U}^{*} \text { if } S_{L} \leq 0 \leq S_{R} \\
\vec{U}_{R} \text { if } S_{R}<0
\end{array}\right.
$$

Here the intermediate state is given by

$$
\vec{U}^{*}=\frac{S_{R} \vec{U}_{R}-S_{L} \vec{U}_{L}-\left(\vec{F}_{R}-\vec{F}_{L}\right)}{S_{R}-S_{L}}
$$


The flux for the HLLE scheme can be written as follows

$$
\vec{F}_{H L L E}\left(\vec{U}_{L}, \vec{U}_{R}\right)=\frac{S^{+} \vec{F}\left(\vec{U}_{L}\right)-S^{-} \vec{F}\left(\vec{U}_{R}\right)}{S^{+}-S^{-}}+\frac{S^{+} S^{-}}{S^{+}-S^{-}}\left(\vec{U}_{R}-\vec{U}_{L}\right)
$$

where

$$
\begin{aligned}
& S^{+}=\max \left\{S_{R}, 0\right\} \\
& S^{-}=\min \left\{S_{L}, 0\right\}
\end{aligned}
$$

Einfeldt estimated the wave speeds for the fastest left and right running waves $\left(S_{L}\right.$ and $\left.S_{R}\right)$ as

$$
\begin{aligned}
& S_{R}=\max \left\{\hat{\lambda}_{2},(u+a)_{R}\right\} \\
& S_{L}=\min \left\{\hat{\lambda}_{3},(u-a)_{L}\right\}
\end{aligned}
$$

where $\hat{\lambda}_{1,2,3}=[\hat{u}, \hat{u}+\hat{a}, \hat{u}-\hat{a}]$ are the Roe-averaged eigenvalues of the inviscid flux Jacobians. This scheme is robust but overly diffusive. In addition, all information about the contact discontinuity is lost. In order to rectify this, Einfeldt ${ }^{9}$ developed the HLLEM scheme. The HLLEM scheme differs from the HLLE scheme by the addition of anti-diffusion terms to the linear characteristic fields as follows

$$
\vec{F}_{H L L E M}\left(\vec{U}_{L}, \vec{U}_{R}\right)=\frac{S^{+} \vec{F}\left(\vec{U}_{L}\right)-S^{-} \vec{F}\left(\vec{U}_{R}\right)}{S^{+}-S^{-}}+\frac{S^{+} S^{-}}{S^{+}-S^{-}}\left(\vec{U}_{R}-\vec{U}_{L}-\sum_{p} \delta^{p} \hat{\alpha}^{p} \hat{R}^{p}\right)
$$

where $\hat{\alpha}^{p}$ are the coefficients associated with the linear degenerated and nonlinear fields for characteristic waves which expand $\vec{U}_{R}-\vec{U}_{L}$ in terms of the right eigenvectors of the Roe averaged flux Jacobians $\hat{R}^{p}$.

$$
\hat{\alpha}^{p}=\hat{L}^{p}\left(\vec{U}_{R}-\vec{U}_{L}\right)
$$

where $\hat{L}^{p}$ is the left eigenvectors of the Roe averaged flux Jacobian. The anti-diffusion coefficients $\delta^{p}$ in Eq. (13) are defined to take out excess numerical dissipation in the linear degenerated fields

$$
\begin{aligned}
& \delta^{1}=\frac{\hat{a}}{|\bar{u}|+\hat{a}} \\
& \delta^{2}=\delta^{3}=0
\end{aligned}
$$

The value of $\bar{u}$ in Eq. (14) is an approximation for the speed of the contact discontinuity. The HLLEM scheme has numerical dissipation of the same order as the Roe scheme. This scheme can also be implemented without using logic statements.

\section{HLLC Scheme}

Toro ${ }^{10}$ developed a two-state approximate Riemann solution that includes the contact discontinuity in the intermediate state. A schematic ( $x-t$ plot) of the HLLC two-state formulation is depicted in Fig. 2. The flux for the HLLC scheme can be written as 


$$
\vec{F}_{H L L C}=\left\{\begin{array}{c}
\vec{F}_{L} \text { if } S_{L}>0 \\
\vec{F}_{L}^{*} \text { if } S_{L} \leq 0<S_{M} \\
\vec{F}_{R}^{*} \text { if } S_{M} \leq 0 \leq S_{R} \\
\quad \vec{F}_{R} \text { if } S_{R}<0
\end{array}\right.
$$

The contact wave speed $S_{M}$ is given by ${ }^{11}$

$$
S_{M}=\frac{\rho_{R} u_{R}\left(S_{R}-u_{R}\right)-\rho_{L} u_{L}\left(S_{L}-u_{L}\right)+p_{L}-p_{R}}{\rho_{R}\left(S_{R}-u_{R}\right)-\rho_{L}\left(S_{L}-u_{L}\right)}
$$

The HLLC scheme will solve both isolated shock and contact waves exactly. The choice of wave speeds also guarantees that the method will be positively conservative ${ }^{12}$. Solutions with this scheme have been found to be comparable to an exact Riemann solver, only with a stronger enforcement of the entropy condition. This scheme does not allow direct access to the anti-diffusion terms. Implementation of the scheme does require logical statements.

\section{HLLE+ Scheme}

Park et al. ${ }^{13,14}$ noted that the form of the HLLEM flux shown in Eq. (12) can be used to specify the HLLE, HLLEM, and Roe fluxes with the appropriate choice of wave speeds $S_{R}$ and $S_{L}$ in Eq. (11) and the contact discontinuity speed $\bar{u}$ in Eq. (14). The schemes can be defined using:

HLLE:

HLLEM:

$$
\begin{aligned}
& S_{R}=\max \left\{\hat{\lambda}_{2},(u+a)_{R}\right\} \\
& S_{L}=\min \left\{\hat{\lambda}_{3},(u-a)_{L}\right\} \\
& |\bar{u}|=\infty
\end{aligned}
$$

$$
\begin{aligned}
& S_{R}=\max \left\{\hat{\lambda}_{2},(u+a)_{R}\right\} \\
& S_{L}=\min \left\{\hat{\lambda}_{3},(u-a)_{L}\right\} \\
& |\bar{u}|=\left|\frac{S_{R}+S_{L}}{2}\right|
\end{aligned}
$$

Roe:

$$
\begin{aligned}
S_{R} & =\hat{\lambda}_{2} \\
S_{L} & =\hat{\lambda}_{3} \\
|\bar{u}| & =|\hat{u}|
\end{aligned}
$$

Park and $\mathrm{Kwan}^{13}$ proposed to combine the wave speed estimates from the HLLEM scheme with the contact velocity estimate from Roe's scheme to form the HLLE+ flux function: 
HLLE+:

$$
\begin{aligned}
& S_{R}=\max \left\{\hat{\lambda}_{2},(u+a)_{R}\right\} \\
& S_{L}=\min \left\{\hat{\lambda}_{3},(u-a)_{L}\right\} \\
& |\bar{u}|=|\hat{u}|
\end{aligned}
$$

Tramel and Nichols ${ }^{12}$ and Park et al. ${ }^{13,14}$ both noted that the anti-diffusion term $\delta^{1}$ is responsible for many of the shortcomings which afflict the HLLE/Roe family of flux functions and used directional pressure sensors to reduce the magnitude of the anti-diffusion term in the presence of strong discontinuities. The directional pressure sensor is calculated along grid lines. The HLLEM scheme or Roe's scheme can be made to transition to the HLLE scheme as the anti-diffusion is reduced. Since the switch is only activated in the vicinity of strong shocks, the switching does not affect other areas of the flow such as boundary layers. This allows hypersonic shocks to be captured without encountering the "carbuncle" phenomena occurring and without corrupting the low numerical dissipation of the scheme in boundary layers. The switch is implemented by defining the contact discontinuity speed $\bar{u}$ as

$$
|\bar{u}|=\beta_{\text {Park }}|\hat{u}|+\left(1-\beta_{\text {Park }}\right) \hat{a}
$$

where the switching function $\beta_{\text {Park }}$ is defined by

$$
\beta_{\text {Park }}=\left\{\begin{array}{l}
1.0 \text { if } k_{p} \leq \Delta_{p} \\
0.0 \text { otherwise }
\end{array}\right.
$$

and the pressure switch sensor $k_{p}$ is defined as

$$
k_{p}=A B S\left(\frac{p_{i+1}-2 p_{i}+p_{i-1}}{p_{i+1}+2 p_{i}+p_{i-1}}\right)
$$

The pressure switch sensor is defined as the average of the sensors in each individual direction when applied to twoor three-dimensional flows. Ref. 13 recommends a value of 0.1 for the threshold value $\Delta_{p}$.

All of the HLLE/Roe family of schemes can be cast in traditional artificial dissipation form given in Eq. (4) with the following choice of eigenvalues:

$$
\begin{aligned}
& \hat{\lambda}_{H L L E, 1}=\left(\left(S^{+}+S^{-}\right) * \hat{u} \quad-2.0 *\left(1-\delta^{1}\right) *\left(S^{+} S^{-}\right)\right) /\left(S^{+}-S^{-}\right) \\
& \hat{\lambda}_{H L L E, 2}=\left(\left(S^{+}+S^{-}\right) *(\hat{u}+\hat{a})-2.0 *\left(1-\delta^{2}\right) *\left(S^{+} S^{-}\right)\right) /\left(S^{+}-S^{-}\right) \\
& \hat{\lambda}_{H L L E, 3}=\left(\left(S^{+}+S^{-}\right) *(\hat{u}-\hat{a})-2.0 *\left(1-\delta^{3}\right) *\left(S^{+} S^{-}\right)\right) /\left(S^{+}-S^{-}\right)
\end{aligned}
$$

The linear eigenvalues in the Roe dissipation matrix transition from

$$
|\hat{u}|=>\frac{1}{2 \hat{a}}\left(\hat{u}^{2}+\hat{a}^{2}\right)
$$

as $\beta_{\text {Park }}$ approaches zero. Alternately, when the Roe-averaged velocity $\hat{u}$ goes to zero the switching function reduces the anti-diffusion from one to 0.5 as $\beta_{\text {Park }}$ approaches zero. This modified contact discontinuity velocity provides enough dissipation to reduce or eliminate "carbuncles" in the vicinity of strong shocks.

\section{Higher Order Spatial Reconstruction}

This effort focuses on extending and upgrading the Roe and HLLC Riemann solvers currently implemented in OVERFLOW 2.15. OVERFLOW 2.1 uses a Monotone Upstream-centered Schemes for Conservation Laws (MUSCL) approach to obtain higher order estimates of the primitive variables for the Riemann inviscid flux 
routines. The code currently includes five options for flux limiters for these MUSCL extrapolations: Koren ${ }^{16,17}$, $\operatorname{minmod}^{18}$, van Albada $^{19}$, WENO ${ }^{20,21}$, and WENOM ${ }^{21,22}$. OVERFLOW 2.1 uses a switch to locally reduce the spatial order of accuracy for the extrapolation for the minmod, van Albada, WENO, and WENOM limiters in the code to first order when strong gradients are encountered. The switch is currently evaluated in each of the computational directions. The switch is defined as

$$
\begin{aligned}
& S_{w_{i}}=\left(\Delta_{s}-1.0\right) * \operatorname{MAX}\left(k_{p}, k_{T}\right) \\
& S_{w_{i}}=\operatorname{MIN}\left(S_{w_{i}}, 1.0\right) \\
& \beta_{\text {old }}=\left(1.0-\operatorname{MAX}\left(S_{w_{i-1}}, S_{w_{i}}, S_{w_{i+1}}\right)\right)^{2}
\end{aligned}
$$

where $\Delta_{s}$ controls the sensitivity of the switch and $\Delta_{s}$ is defined to be greater or equal to one. The parameter $k_{T}$ is a sensor defined using Eq. (23) based on static temperature rather than pressure. The switching function $\beta_{\text {old }}$ ranges from zero to one. Large gradients cause the switching function to reduce to zero locally. Eq. (28) is used to "thicken" the sensor by using neighboring points. The switching function multiplies the higher order contribution to the local variable reconstruction and causes to reduce to first order in space near large discontinuities in pressure or temperature.

\section{OVERFLOW Implementation}

The code has been modified to use a multidimensional switch rather than the current directional switch by taking the maximum value of the Eq. (23) pressure sensor in each computational $(\xi, \eta, \zeta)$ direction. The code currently uses the NAMELIST parameter DELTA to control the sensor sensitivity. The value of the threshold level of the switch $\left(\Delta_{s}\right)$ used in the code is set to 1.0/DELTA. Values of DELTA between 1 and 10 ( $\Delta_{s}$ varying from 1 to 0.1 ) have been found to be adequate to eliminate carbuncles and errors induced when strong shocks are present that are not aligned with the computational grid system. The multidimensional switch is used to locally reduce the spatial order of accuracy of the minmod, van Albada, WENO, and WENOM flux limiters for the Navier-Stokes equations. The new switch is defined as

$$
\beta_{\text {New }}=\left\{\begin{array}{c}
0.0 \text { if } S_{w_{i, j, k}}>1.0 / \text { DELTA } \\
1-\tanh \left(10 \varphi_{p}^{3}\right) \text { otherwise }
\end{array}\right.
$$

where

$$
\varphi_{p}=\operatorname{MAX}\left(S_{w_{i, j, k}} / D E L T A, 0\right)
$$

and $S_{w_{i, j, k}}$ is the maximum of $k_{p}$ for all of the neighboring points in all directions

$$
S_{w_{i, j, k}}=\operatorname{MAX} X_{\substack{l=i-1, i+1 \\ m=j-1, j+1 \\ n=k-1, k+1}}\left(k_{p_{l, m, n}}\right)
$$

The temperature is no longer included in the switching function to eliminate the possibility of large temperature gradients near the wall causing the switching function to activate.

The existing Roe scheme in the code has been modified to become the HLLE+ scheme described in the previous section. The HLLE+ scheme uses the new switching function (Eq. (29)) rather than the switching function of Park (Eq. (22)). The existing HLLC scheme was modified to use the new multidimensional switching function to locally transition to the HLLE scheme using the following flux definition

$$
F_{H L L C+}=\beta_{H L L C+} F_{H L L C}+\left(1-\beta_{H L L C+}\right) F_{H L L E}
$$


where

$$
\beta_{\text {HLLC+ }}=\operatorname{MAX}\left(\beta_{\text {New }}, 0.4\right)
$$

This scheme is similar in spirit to that advocated by Quirk ${ }^{23 .}$ The modified HLLC scheme has been designated HLLC+. The HLLE++ scheme was derived from the HLLE+ scheme by replacing the HLLE+ eigenvalues with a blending of the HLLE+ and a dissipative version of the Roe eigenvalues. The new eigenvalues are defined as

$$
\lambda_{\text {HLLE++ }}=\beta_{\text {New }} \lambda_{H L L E}+\left(1-\beta_{\text {New }}\right) \lambda_{\text {Roe }}
$$

The Roe eigenvalues $\left(\hat{\lambda}_{R o e}\right)$ are defined as

$$
\begin{aligned}
& \lambda_{\text {Roe, } 1}=\operatorname{MAX}(|\hat{u}|, \hat{a}) \\
& \lambda_{\text {Roe }, 2}=\mathrm{H}(|\hat{u}+\hat{a}|, \varepsilon) \\
& \lambda_{\text {Roe, } 3}=\mathrm{H}(|\hat{u}-\hat{a}|, \varepsilon)
\end{aligned}
$$

where $\mathrm{H}$ is the entropy fix of Harten And Hyman ${ }^{24}$ defined as follows

$$
\mathrm{H}(|\lambda|, \varepsilon)=\left\{\begin{array}{lll}
|\lambda| & \text { if } & |\lambda|>\varepsilon \\
\frac{\lambda^{2}+\varepsilon^{2}}{2 \varepsilon} & \text { if } & |\lambda| \leq \varepsilon
\end{array}\right.
$$

and $\varepsilon$ was chosen to be $0.3 *(\lfloor\hat{u}\rfloor+\hat{a})$.

The switching function of Park (Eq. (22)) was found to cause numerical convergence to hang for both the HLLC + and HLLE+ algorithms because of its abrupt transition between states. The old switching function (Eq. (28)) was found to switch too quickly to the more dissipative HLLE algorithm even for small values of the pressure sensor $k_{p}$. The new switching function (Eq. (29)) eliminated both of these problems. A comparison of the new (Eq. (29)), old (Eq. (28)), and Park's (Eq. (22)) switching functions is shown in Fig. 3. DELTA values between five and ten with the new switch should provide similar results to the Park switch.

The relative advantages and disadvantages of the schemes discussed in this paper are summarized as follows:

- Roe

$\checkmark$ Captures stationary grid-aligned shocks exactly

$\times$ Expansion shocks (entropy fix required)

$\checkmark$ Reduced dissipation for boundary layers, etc.

$\checkmark$ Exact capture of stationary contact discontinuities

$\times$ Susceptible to carbuncles

- HLLE

$\checkmark$ Captures stationary grid-aligned shocks exactly

$\checkmark$ No expansion shocks (no entropy fix required)

$\times$ Excessive dissipation for boundary layers, etc.

$\times$ Does not exactly capture of stationary contact discontinuities

$\checkmark$ Not susceptible to carbuncles

- HLLEM

$\checkmark$ Captures stationary grid-aligned shocks exactly

$\checkmark \quad$ No expansion shocks (no entropy fix required)

$\checkmark$ Reduced dissipation for boundary layers, etc.

$\checkmark$ Exact capture of stationary contact discontinuities

- HLLC

$\times$ Susceptible to carbuncles

$\checkmark$ Captures stationary grid-aligned shocks exactly 


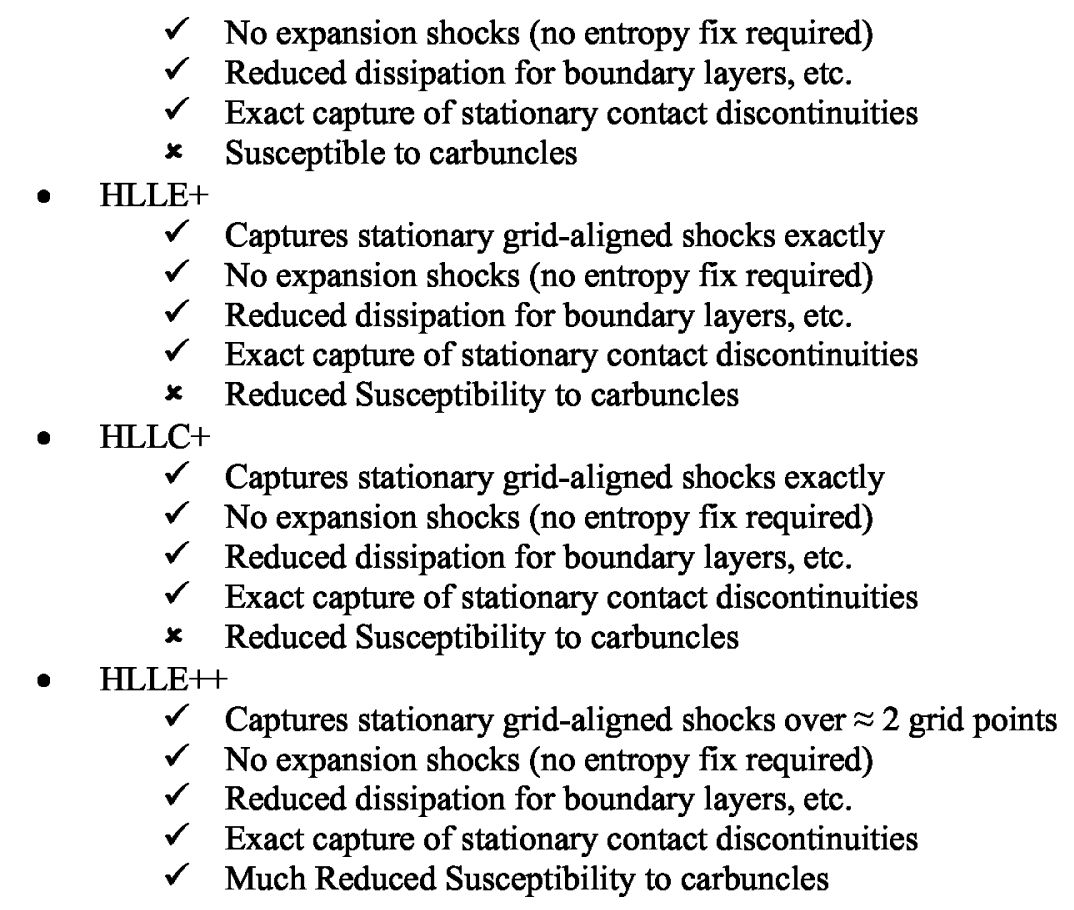

In summary, all of the approximate Riemann algorithms discussed here have been modified to reduce their susceptibility to carbuncles. All of the algorithms use a switch to locally reduce the order of extrapolation to first order in the presence of strong gradients. The HLLC and Roe scheme use a directional switch based on pressure and temperature. The baseline HLLC has no further corrections. The original Roe algorithm also limits the eigenvalues in the anti-diffusion terms to impose an entropy fix to eliminate expansion shocks. The HLLE+ and HLLC+ algorithms use a multidimensional pressure gradient-based switching function to change to the more dissipative HLLE scheme near shocks. The HLLE+ is the HLLE+ scheme with the addition of a limiter invoked by the pressure gradient switch applied to the eigenvalues.

\section{Results}

Solutions using the current OVERFLOW 2.1 HLLC and Roe schemes are denoted as HLLC and Roe in this section. Solutions using the new schemes are denoted as HLLC+, HLLE+, and HLLE++. Note that HLLC+ is exactly the same as HLLC when DELTA=1. Also the HLLE++ scheme is identical to the HLLE+ scheme when $D E L T A=1$. All solutions were run with $3^{\text {rd }}$ order spatial operators unless otherwise noted. The $5^{\text {th }}$ order spatial WENO schemes behave in a similar manner to the $3^{\text {rd }}$ order algorithms.

\section{Inviscid Supersonic Ramp}

The first case demonstrates the problem with the current Riemann solvers in OVERFLOW 2.1 and shows the improvement that result from using the new schemes. The inviscid $M=4.0$ flow over a two-dimensional $30^{\circ}$ ramp is examined. All solutions were performed using the van Albada flux limiter. Fig. 4 shows the 59x219 grid colored by density for the HLLE++ scheme with $D E L T A=5$. Density contours for the HLLC+ algorithm with $D E L T A=1$ are shown in Fig. 5a. The shock is not aligned with the grid. The density contours at the shock are seen to "stair-step" across the grid and errors are produced that are convected downstream of the shock. Similar results were obtained with the HLLE+ and HLLE++ algorithms with $D E L T A=1$ and for the Roe and HLLC algorithms with all DELTA values. Density contours for the HLLC+ algorithm with $D E L T A=5$ are shown in Fig. $5 \mathrm{~b}$. The error streaks behind the shock are significantly reduced. Similar results were obtained with the HLLE+ algorithm. Density contours for the HLLE++ algorithm with $D E L T A=5$ are shown in Fig. $5 \mathrm{c}$. The error streaks are almost completely removed by this algorithm.

The density on the comparison line indicated in Fig. 5 are shown for all five algorithms with $D E L T A=1,5,10$, and 20 in Fig. 6. All of the algorithms display at least some of the convected error that is originating from the shock when DELTA=1. Increasing DELTA has no effect on reducing the error with the Roe or HLLC algorithms. 
Increasing DELTA is shown to greatly reduce the erroneous wiggles for the HLLE+ and HLLC+ algorithms. The HLLE+ + algorithm decreases the error even further when DELTA is increased. These results show that the postshock oscillations are a result of the multi-dimensional resolution of the shock since the error is significantly reduced with the use of a multidirectional switch.

It has often been conjectured that reducing the spatial order of accuracy could remove the error associated with the misaligned shock through the addition of large amounts of numerical dissipation. The density on the comparison line for all algorithms using first order spatial algorithms is shown in Fig. 7. The density oscillations are reduced compared to the $3^{\text {rd }}$ order spatial results for all algorithms, but the error is still much larger than that seen for the $3^{\text {rd }}$ order spatial HLLC+, HLLE+, and HLLE++ algorithms with DELTA $>1$. Hence simply reducing to a first order spatial algorithm at the shock will reduce but will not eliminate the error generated at the shock.

The minimum oscillation defined in terms of the difference of the maximum and minimum density divided by the average density for each algorithm is shown in Table 1 . The HLLE++ algorithm produces the lowest density oscillation. The average value of the density for the HLLC+, HLLE+, and HLLE++ algorithms are in reasonable agreement with the theoretical density behind the shock $\left(\rho / \rho_{\infty}=3.703\right)$. The HLLC+, HLLE+, and HLLE++ algorithms all produce results with lower density oscillations and better agreement with the theoretical density than the central difference algorithm.

\begin{tabular}{|c|c|c|c|}
\hline Algorithm & $\boldsymbol{D E L T A}$ & $\boldsymbol{\rho} / \boldsymbol{\rho}_{\infty}$ & $\Delta \rho / \rho_{\text {avg }}(\%)$ \\
\hline Roe & 10 & 3.682 & 17.19 \\
\hline HLLC & 10 & 3.679 & 22.77 \\
\hline HLLC+ & 20 & 3.684 & 0.573 \\
\hline HLLE+ & 20 & 3.683 & 0.706 \\
\hline HLLE++ & 20 & 3.683 & 0.360 \\
\hline
\end{tabular}

Table 1. Minimum density oscillation for each algorithm for the $M=430^{\circ}$ ramp.

The solutions were run using grid sequencing with 100 iterations on the coarse mesh, 100 iterations on the medium grid, and finally 1000 iterations on the fine grid. The convergence history for the Roe and HLLE++ algorithms is shown in Fig. 8. The HLLC convergence history is similar to the Roe algorithm. The HLLC+ and HLLE+ convergence history is similar to the HLLE++ results. The HLLE++ algorithm with DELTA $>1$ has much better convergence properties than the algorithm with $D E L T A=1$. The Roe and HLLC algorithm convergence does not improve with increasing values of DELTA.

The switching function $\beta_{\text {New }}$ is shown in Fig. 9 for the HLLE++ algorithm with DELTA=5. The switching function rapidly approaches zero in the vicinity of the shock. The HLLC+ algorithm is equivalent to the HLLC algorithm in smooth regions of the flow, but the HLLC+ algorithm switches to the HLLE scheme near shocks where the switching function is zero. Thus it follows that the HLLE scheme is the agent for removing the error associated with the non-grid aligned shock for this case.

\section{Inviscid Supersonic Ramp with Channel}

The second case builds upon the first case with the addition of shock-shock interaction. The inviscid $\mathrm{M}=3.0$ flow over a two-dimensional $30^{\circ}$ ramp in a channel is examined. The channel height is twice the ramp height. All solutions were performed using the van Albada flux limiter. Fig. 10 shows the 95x101 grid colored by density for the HLLE++ scheme with $D E L T A=5$. Density contours for the HLLC+ algorithm with $D E L T A=1$ are shown in Fig. 11a. The shock is not aligned with the grid. The density contours are seen to possess streaks downstream of the oblique and normal shocks. Similar results were obtained with the HLLE+ and HLLE++ algorithms with $D E L T A=1.0$ and for the HLLC algorithm with all $D E L T A$ values. No steady state solution could be obtained for the Roe algorithm with $D E L T A=1$. Density contours for the HLLC+ algorithm with $D E L T A=5$ are shown in Fig. $11 \mathrm{~b}$. The density contours behind the oblique shock are greatly improved, but there are still nonphysical density oscillations behind the normal shock. Similar results were obtained with the HLLE+ algorithm. Density contours for the HLLE++ algorithm with $D E L T A=5$ are shown in Fig. 11c. There is no visible density streaking or oscillations present behind either the normal or oblique shock.

The density on the comparison line indicated in Fig. 11 are shown for all five algorithms with $D E L T A=1,5,10$, and 20 in Fig. 12. All of the algorithms display at least some of the convected error that is originating from the shock when $D E L T A=1$. Increasing DELTA has no effect on reducing the error with the Roe or HLLC algorithms. Increasing DELTA is shown to greatly reduce the erroneous wiggles for the HLLE+ and HLLC+ algorithms. The 
HLLE+ + algorithm eliminates the error when DELTA is increased. All of the algorithms show differences in the expansion region when DELTA is increased.

The solutions were run using grid sequencing with 100 iterations on the coarse mesh, 100 iterations on the medium grid, and finally 1000 iterations on the fine grid. The convergence history for the HLLC and HLLE++ algorithms is shown in Fig. 13. The Roe convergence history is similar to the HLLC algorithm. The HLLC+ and HLLE+ convergence history is similar to the HLLE++ results. The HLLE++ algorithm with DELTA 1 has much better convergence properties than the algorithm with DELTA $=1$. The Roe and HLLC algorithm convergence improves some with increasing values of DELTA.

The switching function $\beta_{\text {New }}$ is shown in Fig. 14 for the HLLE++ algorithm with $D E L T A=5$. The switching function rapidly approaches zero in the vicinity of the shock. The switch approaches zero in the expansion region by the ramp upper corner.

\section{Shock Tube}

Shock tube problems have long been used as test cases for unsteady flows and are used here to demonstrate that the algorithms are time accurate when DELTA $>1$. The position of the shock, expansion, and contact discontinuity in inviscid flow can be predicted theoretically. The conditions chosen for this problem are:

Left state: $\rho / \rho_{\infty}=1.0, \mathrm{p} / \mathrm{p}_{\infty}=1.0$

Right state: $\rho / \rho_{\infty}=0.1, \mathrm{p} / \mathrm{p}_{\mathrm{s}}=0.1$

The results are evaluated at the nondimensional time of 0.2 (320 iterations for the nondimensional time step of 0.000625 ). The solution was run using 401 grid points with ten Newton subiterations per time step. The density at $t=0.2$ for each algorithm is shown in Fig. 15. The solutions are almost indistinguishable and are in good agreement with theory for all DELTA values.

\section{Inviscid Isentropic Vortex Convection}

The ability to conserve both the vortex shape and strength is important in many unsteady cases in which a shed vortex interacts with bodies well downstream of the vortex origin. The following test case ${ }^{25}$ can be used to examine the relative level of numerical dissipation and dispersion for inviscid flux algorithms. An isentropic vortex of nondimensional strength $\Gamma=5$ is centered on a uniform grid of size 10 units by 10 units. A $101 \times 101$ grid $(\Delta x=0.1)$ was used for most of the results presented here. The vortex is allowed to convect downstream at free stream Mach number of 0.5 with a non-dimensional time step $\left(d t \frac{U_{\infty}}{L_{\operatorname{Re} f}}\right)$ of 0.01 . The velocity, temperature, density, and pressure for the vortex are given by

$$
\begin{aligned}
& u=u_{\infty}-\frac{\Gamma}{2 \pi}\left(z-z_{0}\right) \exp \left[0.5\left(1-\bar{R}^{2}\right)\right] \\
& w=\frac{\Gamma}{2 \pi}\left(x-x_{0}\right) \exp \left[0.5\left(1-\bar{R}^{2}\right)\right] \\
& T=T_{\infty}-\frac{(\gamma-1) \Gamma^{2}}{8 \gamma \pi} \exp \left(1-\bar{R}^{2}\right) \\
& \rho=T^{\frac{1}{\gamma-1}} \\
& p=\rho^{\gamma}
\end{aligned}
$$

where $\bar{R}^{2}=\left(x-x_{0}\right)^{2}+\left(z-z_{0}\right)^{2}$ and $x_{0}$ and $z_{0}$ define the location of the vortex center at any given point in time. Here $\gamma$ is the ratio of specific heats. The grid is given periodic boundary conditions in the flow direction. The vortex should complete one cycle on the grid every 1000 time steps. All calculations used $2^{\text {nd }}$ order time and three Newton subiterations. The vortex is allowed to convect through the grid five times. The grid and the starting vortex can be seen in Fig. 16. 
The effect of spatial order of accuracy on the minimum density of the vortex for the HLLC scheme is shown for reference in Fig. 17. Note the dramatic difference in dissipation level between $1^{\text {st }}$ and $2^{\text {nd }}$ order space for this case. The minimum density for the vortex with all of the flux schemes and varying DELTA is shown in Fig. 18. The Roe and HLLC schemes remain $3^{\text {rd }}$ order in space for all values of DELTA. The HLLC+, HLLE+, and HLLE++ algorithms are relatively insensitive to the value of $D E L T A$, with both algorithms reducing to about second order for $D E L T A=20$. The HLLC+, HLLE+, and HLLE++ algorithms remain near $3^{\text {rd }}$ order in the preferred range of $1 \leq$ $D E L T A \leq 10$ shown to adequately remove the error associated with non-grid aligned shocks for the previous cases.

\section{Hypersonic Cylinder Bow Shock}

The hypersonic bow shock experiment data of Holden, et. $\mathrm{al}^{26}$ provides a good test of the ability of the numerical schemes to capture extremely strong shocks and to predict heat transfer at high speeds. The experiment was run at a free stream Mach number of 16.01, a Reynolds number (based on cylinder diameter) of $9.11 \times 10^{4}$, a free stream temperature of $77.8^{\circ} \mathrm{R}$, and a wall temperature of $540^{\circ} \mathrm{R}$. The nitrogen test medium can still be considered a perfect gas at these conditions. The flow over the 3 in. diameter cylinder is laminar for this case. This case has a temperature ratio across the shock of about 50 and a pressure ratio across the shock of about 300 . Two computational grids were used in this study. Both grids had $101 \times 101$ with a wall spacing corresponding to $\mathrm{y}^{+}$value of 0.1 . No effort was made to align the grid with the shock for one of the grids. The bow shock in the nose region was aligned with the grid for the second mesh.

The flow field was initialized with a free stream Mach number of 16.01. Two levels of grid sequencing were used to set up the flow field (50 iterations on the coarse level and 50 iterations on the mid level). The case was run time accurately with a time step of $2.9 \times 10^{-5} \mathrm{sec}$. Three Newton subiterations were used at each time step with all of the inviscid flux algorithms. The grid and temperature contours for both grids are shown in Fig. 19.

Temperature contours using the HLLC+ algorithm with $D E L T A=1$ and 5, and the HLLE++ algorithm with $D E L T A=5$ are shown in Fig. 20. The contours on the centerline behind the shock are slightly different between the $D E L T A=1$ and $D E L T A=5$ results. There are further differences as the flow inside the shock proceeds around the cylinder. The HLLE++ algorithm with $D E L T A=5$ produces much smoother and rounder contours than the HLLC + algorithm. The Roe, HLLC, and HLLE+ results are similar to the HLLC+ results.

Predicted pressure coefficient is compared to modified Newtonian theory for the HLLE++ scheme in Fig. 21. All of the algorithms produced similar results and are in good agreement with theory in the nose region where the theory is valid. Predicted heat transfer is compared to data in Fig. 22 for the HLLE+ scheme. All of the flux algorithms produced similar results and were in good agreement with the data. The stagnation heat transfer is slightly higher when $D E L T A=1$ on the shock aligned grid for all the algorithms. The stagnation heat transfer is slightly lower when $D E L T A=1$ on the non-shock aligned grid. All of the results are well within the scatter of the data. The solutions for DELTA $>1$ all predict about the same level of heat transfer. Since all of the algorithms show the similar behavior in peak heat transfer, this can be attributed to the reduction of order of the flux limiters in the vicinity of the shock and not to the switching to the HLLE scheme.

The L2 norm of the residuals is shown in Fig. 23 for the HLLE++ algorithm on both grids. Convergence is improved with increasing DELTA for this algorithm. The solution converges an order of magnitude further on the shock aligned grid. Similar behavior was noted for all algorithms. The switching function $\beta_{N e w}$ is shown in Fig. 24 for $D E L T A=5$. The switching function is one except near the shock and at the downstream boundaries.

\section{Hypersonic Double Cone}

The hypersonic double cone experiments of Holden and Wadhams ${ }^{27}$ provides a very demanding test of the ability of the code to predict the heat transfer at high speeds. The geometry for this case is shown in Fig. 25. Details of the flow field are shown in Fig. 26. The attached shock from the first cone interacts strongly with the detached shock associated with the second cone. This shock/shock interaction produces a transmitted shock which in turn impinges on the surface of the second cone. This impingement produces very high surface heat transfer rates and pressures. In addition, the high pressures which result at the cone-cone junction cause the flow to separate in this region. This separation bubble in turn interacts with the inviscid flow field, which impacts the strength of the transmitted shock, etc. and the entire flow field is very dependent on the strengths of the relative interactions. Computational predictions of this type of flow are very sensitive to numerics, grid resolution, etc. (Druget, Candler, and Nompelis ${ }^{28}$ ).

The computational results are compared to the experimental results of Holden's Run 35. The experiment was run at a free stream Mach number of 12.06 , a Reynolds number of $6.79 \times 10^{4}$ per foot, a free stream temperature of $182^{\circ} \mathrm{R}$ and a wall temperature of $533^{\circ} \mathrm{R}$. The test medium was pure nitrogen and is considered a perfect gas at these conditions. The flow conditions were chosen such that the flow over the entire length of the cone remains laminar. 
Computations were run on a $305 \times 129$ grid. This grid is one level coarser than the medium grid used in Ref. 15 . The grid colored by temperature is shown in Fig. 27. The bow shock on the second cone is not aligned with the grid.

Temperature contours are shown in Fig. 28. The HLLC+ DELTA=1 and HLLC+ DELTA $=5$ results are similar. Both of these cases show some "stair-stepping" behind the bow shock on the second cone with error that is convected downstream of the shock. The HLLE+ + contours are much cleaner in this region of the flow. The Roe, HLLC, and HLLE+ results are similar to the HLLC+ contours.

The surface pressure coefficient is shown for all the algorithms in Fig. 29. The surface heat transfer is shown in Fig. 30. The DELTA=1 results compare the best with the data for all algorithms. The Roe and HLLC results are very sensitive to the value of DELTA. The HLLC+, HLLE+, and HLLE++ results are less sensitive to DELTA for $D E L T A \leq 10$ than the Roe and HLLC algorithms, but a dependency still exists. The separation region at the intersection of the two cones decreases as DELTA is increased causing the surface pressure coefficient and heat transfer predictions to move away from the data. The final expansion region following the end of the second cone shows a large dependency on DELTA for all algorithms. This dependency can be attributed to the switching function and indicates that using values of DELTA between one and five would be preferable for this case.

The switching function $\beta_{\text {New }}$ is shown in Fig. 31 for DELTA=5. The switching function is less than one in the vicinity of the shock, at the upper corner at the start of the expansion region, and at the start and end of the separation region at the intersection of the two cones. Convergence for the Roe and HLLE++ algorithms are shown in Fig. 32. Both of these algorithms indicate convergence plateaus with a higher error level for DELTA=20.

\section{Capsule}

The final case is a three dimensional capsule geometry shown in Fig. 33. The flight conditions were a Mach number of $4.02,16^{\circ}$ angle-of-attack, a free stream temperature of $342^{\circ} \mathrm{R}$, and a Reynolds number of $2.49 \times 10^{5}$ per inch. The ratio of specific heats was 1.246. The walls were treated using adiabatic no-slip boundary conditions. Two grid systems with $7.3 \times 10^{6}$ points were used in this study and are shown in Fig. 34. One grid was aligned with the bow shock while the other grid was not aligned with the shock. The non-shock aligned grid is representative of the grid required for a moving body simulation where the shock will move during the simulation.

Mach number contours on the symmetry plane for the shock aligned grid are shown in Fig. 35 for the HLLC+ scheme with $D E L T A=1$, HLLC+ scheme with $D E L T A=5$ and the HLLE+ scheme with DELTA=5. The HLLC+ scheme with $D E L T A=1$ shows carbuncle structures along the bow shock. All of the schemes had similar carbuncles when DELTA=1. The Roe and HLLC algorithms produced carbuncles for all values of DELTA. The HLLC+, HLLE+, and HLLE++ algorithms all produced reasonable results for DELTA $>1$. Mach number contours on the symmetry plane for the non-shock aligned grid are shown in Fig. 36 for the HLLC+ scheme with DELTA=1, HLLC+ scheme with $D E L T A=5$ and the HLLE++ scheme with $D E L T A=5$. The HLLC+ scheme with DELTA=1 shows carbuncle structures along the bow shock. All of the schemes had similar carbuncles when $D E L T A=1$. The Roe and HLLC algorithms produced carbuncles for all values of DELTA. The HLLC+ and HLLE+ algorithms have a small amount of error from the shock "stair-stepping" in the region behind the bow shock for $D E L T A>1$. The HLLE++ algorithm predicts a flow field that is almost identical to the shock-aligned grid results for DELTA $>1$.

Pressure coefficient distributions on the symmetry plane are shown in Fig. 37 for the shock-aligned grid and in Fig. 38 for the non-shock aligned grid. The distributions show the same trends as seen in the Mach number contours. A rise in pressure is seen at about $Z=-0.6$ on the windward side for the non-shock aligned grid. This pressure rise is associated with a disturbance in the bow shock. Fig. 39 shows the pressure coefficient on the symmetry plane for the HLLC+ algorithm with $D E L T A=5$ and the HLLE++ algorithm with $D E L T A=5$ on both grids. All of the solutions are in agreement except for the HLLC+ result with $D E L T A=5$ on the non-shock aligned grid.

The switching function $\beta_{\text {New }}$ is shown in Fig. $\mathbf{4 0}$ for DELTA=5 for both grids. The switching function behaves similarly for both grids. The switching function is less than one in the bow shock region and in the vicinity of the weaker shocks on the leeward side of the capsule

\section{Conclusions}

Traditional approximate Riemann solvers such as the HLLC or Roe algorithms in OVERFLOW 2.1 do not perform well when the grid is not aligned with strong shocks in the flow field. Numerical error in the form of "carbuncles" or "stair-steps" is formed in the vicinity of the shock. This error is convected downstream and reduces the accuracy and stability of the solution. In high speed moving body simulations and complex flow fields it is often impossible to modify the grid to maintain shock alignment.

Four approaches for removing the non-grid aligned shock error. These approaches include adding entropy fixes, locally reducing to first order in space in the shock region, modifying the anti-diffusion terms of the flux, and locally 
switching to a more dissipative algorithm near the shock. None of these fixes by themselves were capable of eliminating the error introduced by non-grid aligned shocks. The HLLE++ scheme includes all of the approaches except for the entropy fix and was found to perform better than any of the other algorithms investigated.

The HLLC+, HLLE+, and HLLE++ algorithms were shown to provide significantly better solutions than the original Roe or HLLC algorithms for problems with strong shocks that are not aligned with the grid. This is due to the fact that the HLLC+, HLLE+, and HLLE++ use a pressure gradient based switching function to locally transition to the more dissipative HLLE algorithm in the vicinity of strong shocks. The results here also indicate that the use of a temperature sensor in addition to the pressure sensor is not required to obtain stable and accurate solutions with large flow field discontinuities. The temperature sensor may be invoked in the boundary layer, while the pressure sensor would not be invoked near the wall.

The HLLC+, HLLE+, and HLLE++ were also shown to maintain time accuracy and provide accurate heat transfer. The new algorithms were also shown to provide low numerical dissipation for the convecting vortex case for the range of DELTA values required to remove error in the vicinity of non-grid aligned shocks. The HLLE++ algorithm produced almost grid independent solutions regardless of grid alignment with strong shocks. Numerical convergence was improved for all of the new algorithms for $1>D E L T A \leq 10$.

The switching function was shown to affect expansion regions as well as shocks in the example cases. This is not a desirable feature. Fortunately low values of the DELTA input parameter $(1<D E L T A \leq 5)$ were found to be sufficient to minimize the formation of the numerical error near the shock for this algorithm. The lower values of DELTA will also assure that the algorithm maintains low numerical dissipation. Hence it is recommended that smaller values of DELTA be used in applications with the HLLC+, HLLE+, or HLLE++ algorithms.

The additional eigenvalue limiting used in the HLLE+ scheme consistently produced the best results for all the problems investigated here. The eigenvalue limiting in the HLLE+ scheme tends to smear the shock over two cells. The HLLC+ and HLLE+ schemes are capable of capturing the shock in one cell. Based on the results here, the accuracy and stability of the results more than offset the slight smearing of the shock with the HLLE++ scheme.

All of the examples in this paper used the $3^{\text {rd }}$ order spatial MUSCL extrapolation to reach higher order. The $5^{\text {th }}$ order WENO extrapolations used in OVERFLOW 2.1 have similar sensitivity to carbuncles and "stair-stepping" as the $3^{\text {rd }}$ order spatial MUSCL schemes. The WENO schemes show the same improvements when used in conjunction with the HLLC+, HLLE+, or HLLE++ algorithms.

\section{References}

${ }^{1}$ Roe, P., “Approximate Riemann Solver, Parameter Vectors and Difference Schemes," J. Comput. Phys., Vol. 43, 1981, pp. 357-372.

${ }^{2}$ Harten, A., Lax, P., van Leer, B., "On Upstream Differencing and Godunov-type Schemes for Hyperbolic Conservations Laws," SIAM Review, Vol. 25, 1983, pp. 35-61.

${ }^{3}$ Yee, H., "Upwind and Symmetric Shock-Capturing Schemes," NASA-TM-89464, 1987.

${ }^{4}$ Muller, B., "Simple Improvements of an Upwind TVD Scheme for Hypersonic Flow," AIAA-89-1977, 1989.

${ }^{5}$ Lin, H., "Dissipation Addition to the Flux-Difference Splitting," J. Comput. Phys., Vol. 117, 1995, pp. $20-27$.

${ }^{6}$ Candler, G., Barnhardt, M., Drayna, T., Nompelis, I., Peterson, D., and Subbereddy, P., "Unstructured Grid Approaches for Accurate Aeroheating Simulations," AIAA-2007-3959, 2007.

${ }^{7}$ Kandula, M. and Buning, P., "Implementation of LU-SGS Algorithm and Roe Upwinding Scheme in OVERFLOW Thin-Layer Navier-Stokes Code," AIAA-94-2357, Jun. 1994.

${ }^{8}$ Einfeldt, B., "On Godunov Methods for Gas Dynamics," SIAM Journal of Numerical Analysis, Vol.25, No. 2, April 1988, pp. 294-318.

${ }^{9}$ Einfeldt, B., Munz, C., Roe, P., and Sjogreen, B., "On Godunov-type Methods Near Low Densities," J. Comput. Phys., Vol. 92, 1991, pp. 273-295.

${ }^{10}$ Toro, E., Spruce, M., and Speares, W., "Restoration of the Contact Surface in the HLL Riemann Solver," Shock Waves, Vol. 4, 1994, pp. 25-34.

${ }^{11}$ Batten, P., Clarke, N., Lambert, C., and Causon, D., "On the Choice of Wavespeeds for the HLLC Riemann Solver," SIAM J. Sci. Comput., Vol. 18, No. 6, Nov. 1997, pp. 1553-1570.

${ }^{12}$ Tramel, R., and Nichols, B., "A Highly Efficient Numerical Method for Overset Mesh Moving-Body Problems," AIAA Paper No. 97-2040, 1997.

${ }^{13}$ Park, S.-H., and Kwon, J., “An Improved HLLE Method for Hypersonic Viscous Flows," AIAA-2001-2633, Jun. 2001.

${ }^{14}$ Park, S.-H., Lee, J., and Kwon, J., "Preconditioned HLLE Method for flows at all Mach Numbers," AIAA 
Journal, Vol. 44, No. 11, Nov. 2006, pp. 2645-2653.

${ }^{15}$ Nichols, R., Tramel, R., and Buning, P., "Solver and Turbulence model Upgrades to OVERFLOW 2 for Unsteady and High-Speed Flow Applications," AIAA-2006-2824, Jun. 2006.

${ }^{16}$ Koren, B., "Upwind Schemes, Multigrid and Defect Correction for the Steady Navier-Stokes Equations," Proceedings of the $11^{\text {th }}$ International Conference on Numerical Methods in Fluid Dynamics, edited by D. Dwoyer, M. Hussani, and R. Vougt, Springer-Verlag, Berlin, 1989.

${ }^{17}$ Srinivasan, G., Baeder, J., Obayashi, S., and McCroskey, W., "Flowfield of a Lifting Rotor in Hover: A NavierStokes Solution," AIAA Journal, Vol. 30, No. 10, Oct. 1992, pp. 2371-2378.

${ }^{18}$ Hirsch, C., Numerical Computation of Internal and External Flows: Volume 2. Wiley-Interscience, 1988.

${ }^{19}$ van Albada, G. D., Van Leer, B., and Roberts, W., W., "A Comparative Study of Computational Methods in Cosmic Gas Dynamics," Astronomy and Astrophysics, Vol. 108, 1982 pp. 76-84.

${ }^{20}$ Titarev, V. and Toro, E., "Finite-Volume WENO Schemes for Three-Dimensional Conservation Laws," J. of Com. Phys., Vol. 201, 2004, pp. 238-260.

${ }^{21}$ Nichols, R., Tramel, R., and Buning, P., "Evaluation of Two High Order WENO Schemes," AIAA-2007-3920, Jun. 2007.

${ }^{22}$ Henrick, A., Aslam, T., and Powers, J., "Mapped Weighted Essentially Non-oscillatory Schemes: Achieving Optimal Order Near Critical Points," J. of Comput. Phys., Vol. 207, 2005, pp 542-567.

${ }^{23}$ Quirk, J. J., "A Contribution to the Great Riemann Solver Debate". Int. J. Numer. Meth. Fluids Vol. 18, 1994, pp. 555-574.

${ }^{24}$ Harten, A., and Hyman, J. M., "Self-Adjusting Grid for One-Dimensional Hyperbolic Conservation Laws" $J$. Comp. Phys. Vol. 50, No. 2, 1983, pp. 235-269.

${ }^{25}$ Yee, H-C., Sandham, N., and Djomehri, M., "Low Dissipative High Order Shock-Capturing Methods using Characteristic-Based Filters", J. Comput. Phys. Vol. 150, 1999, pp. 199-238.

${ }^{26}$ Holden, S. M., Kolly, J. M., and Martin, S. C., "Shock/Shock Interaction Heating in Laminar and Low-Density Hypersonic Flows," AIAA-96-1866, Jun. 1996.

${ }^{27}$ Holden, M. S., and Wadhams. T. P., "Code Validation Study of Laminar Shock/Boundary Layer and Shock/Shock Interactions in Hypersonic Flow. Part A: Experimental Measurements," AIAA-2002-1031, Jan. 2002.

${ }^{28}$ Druget, M.C., Candler, G. V., and Nompelis, I. N., "Simulations of Viscous Hypersonic Double-Cone Flows: Influence of Numerics," AIAA-2003-3548, Jun. 2003. 


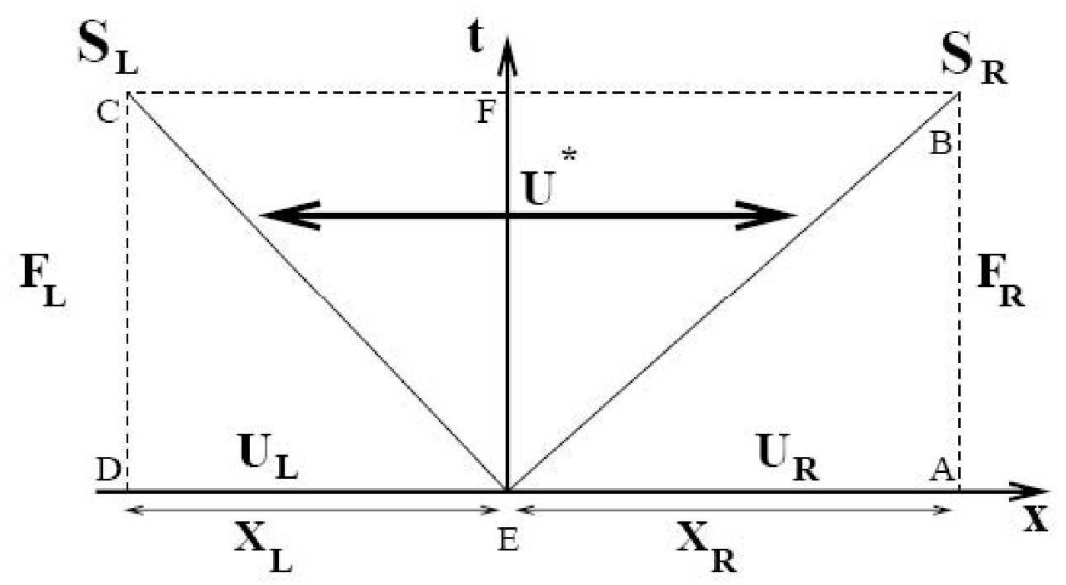

Figure 1. Simplified Riemann fan for the HLL scheme with one intermediate state.

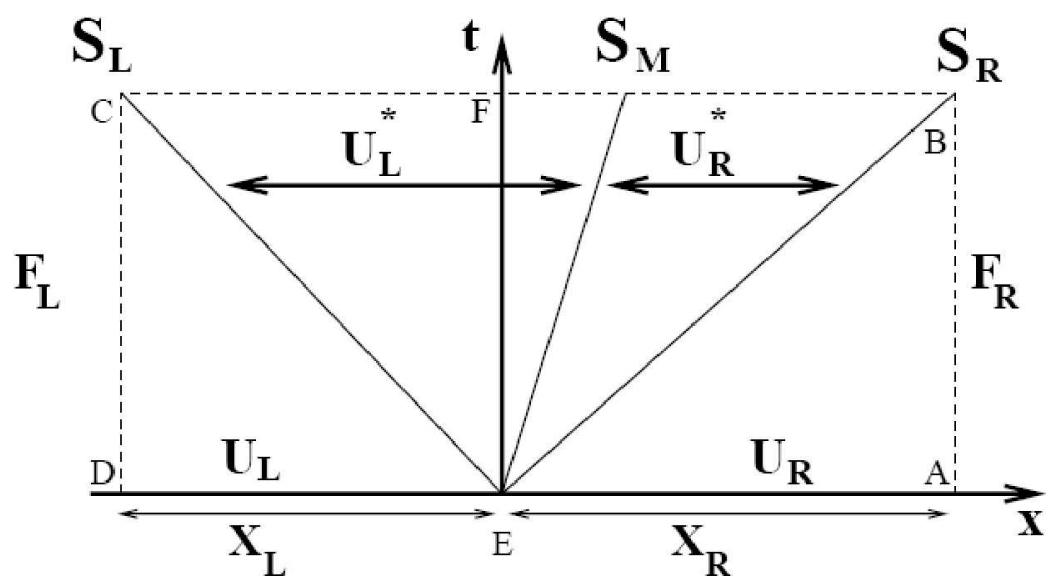

Figure 2. Simplified Riemann fan for the HLLC scheme with two intermediate states.
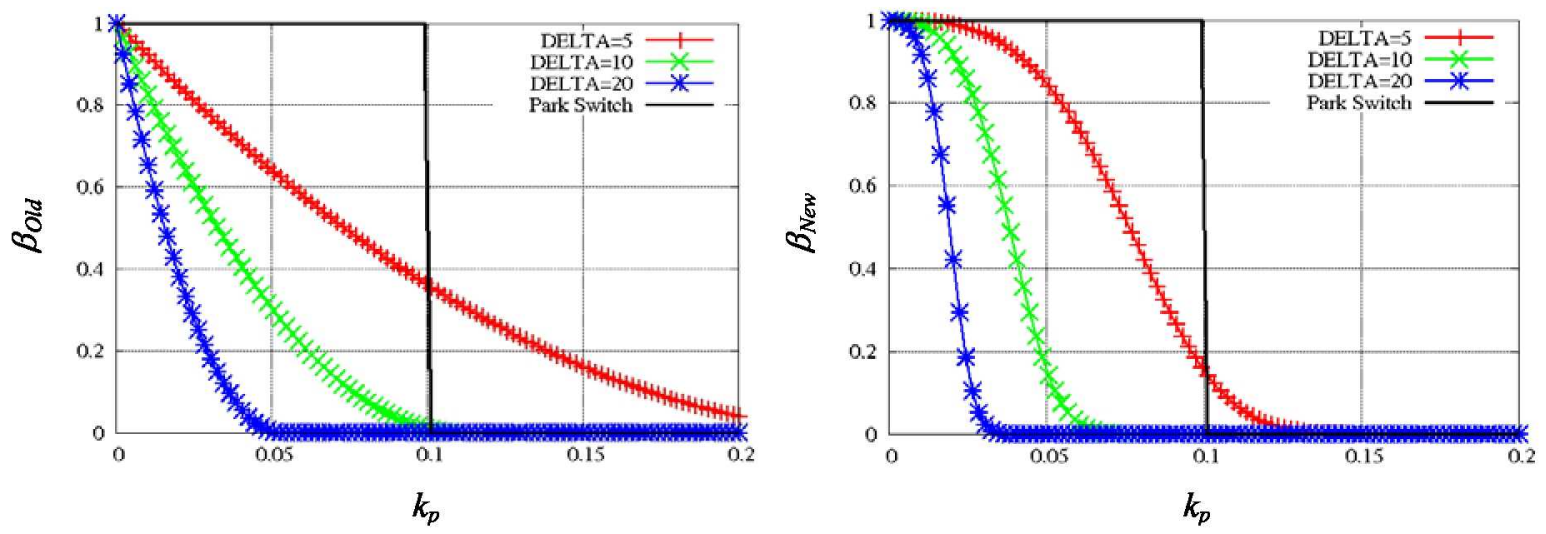

Figure 3. Switching functions for the HLLC+ and HLLE+ algorithms. 


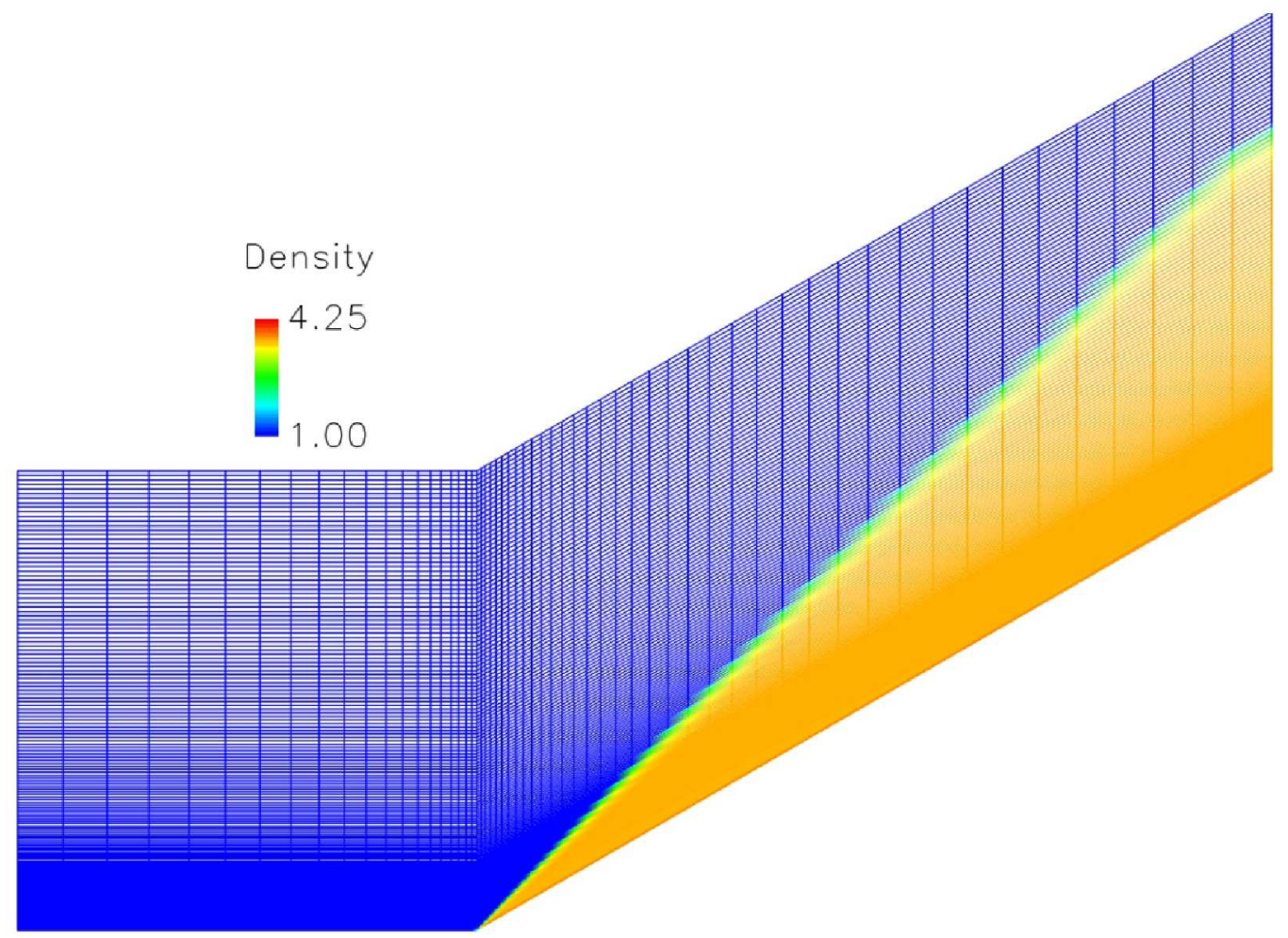

Figure 4. Grid colored by density for the $M=4.030^{\circ}$ ramp.

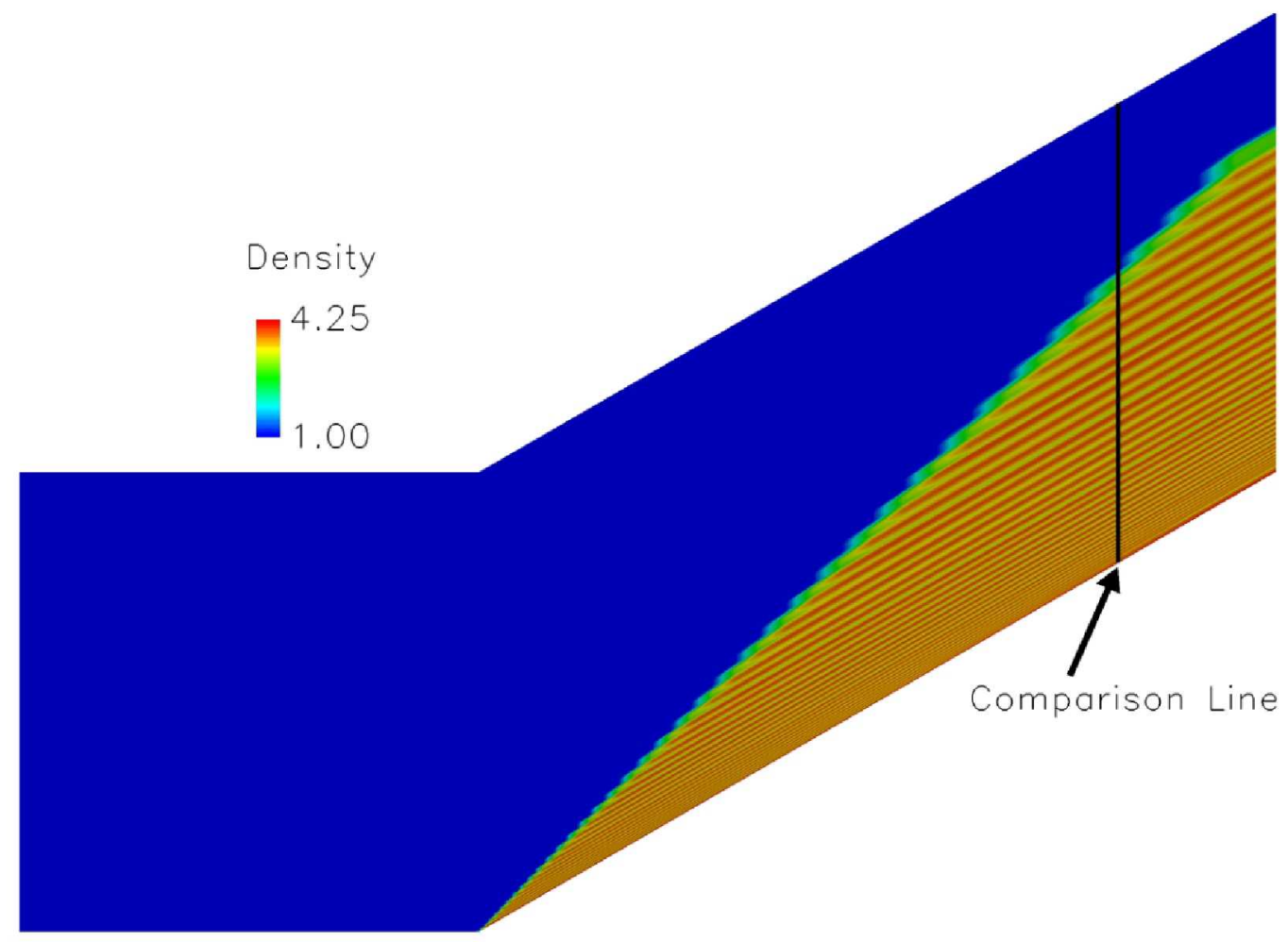

Figure 5a. Density contours using HLLC + with $D E L T A=1$ for the $M=4.030^{\circ} \mathrm{ramp}$. 


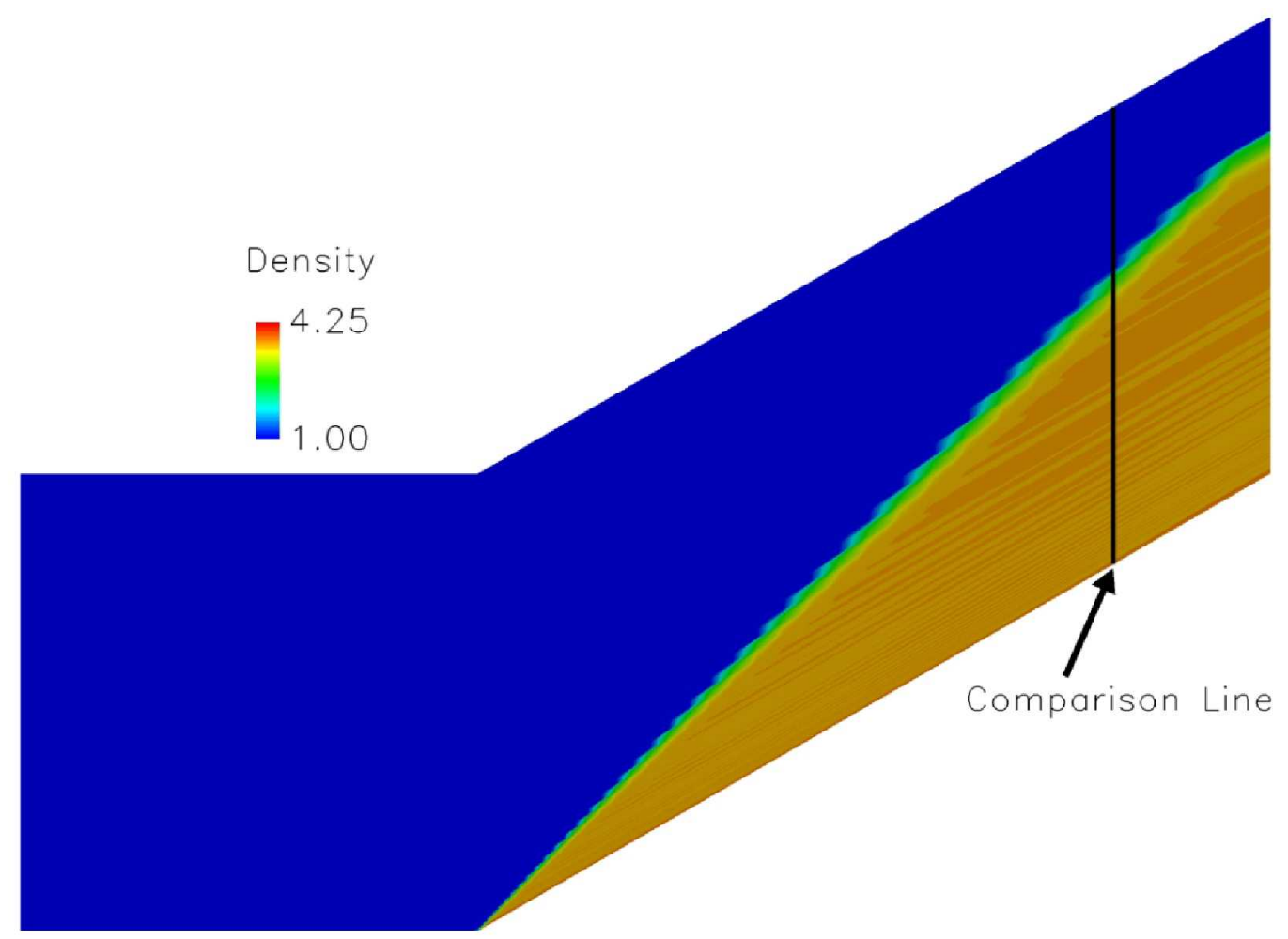

Figure $5 \mathrm{~b}$. Density contours using HLLC + with $D E L T A=5$ for the $\mathrm{M}=4.030^{\circ} \mathrm{ramp}$.

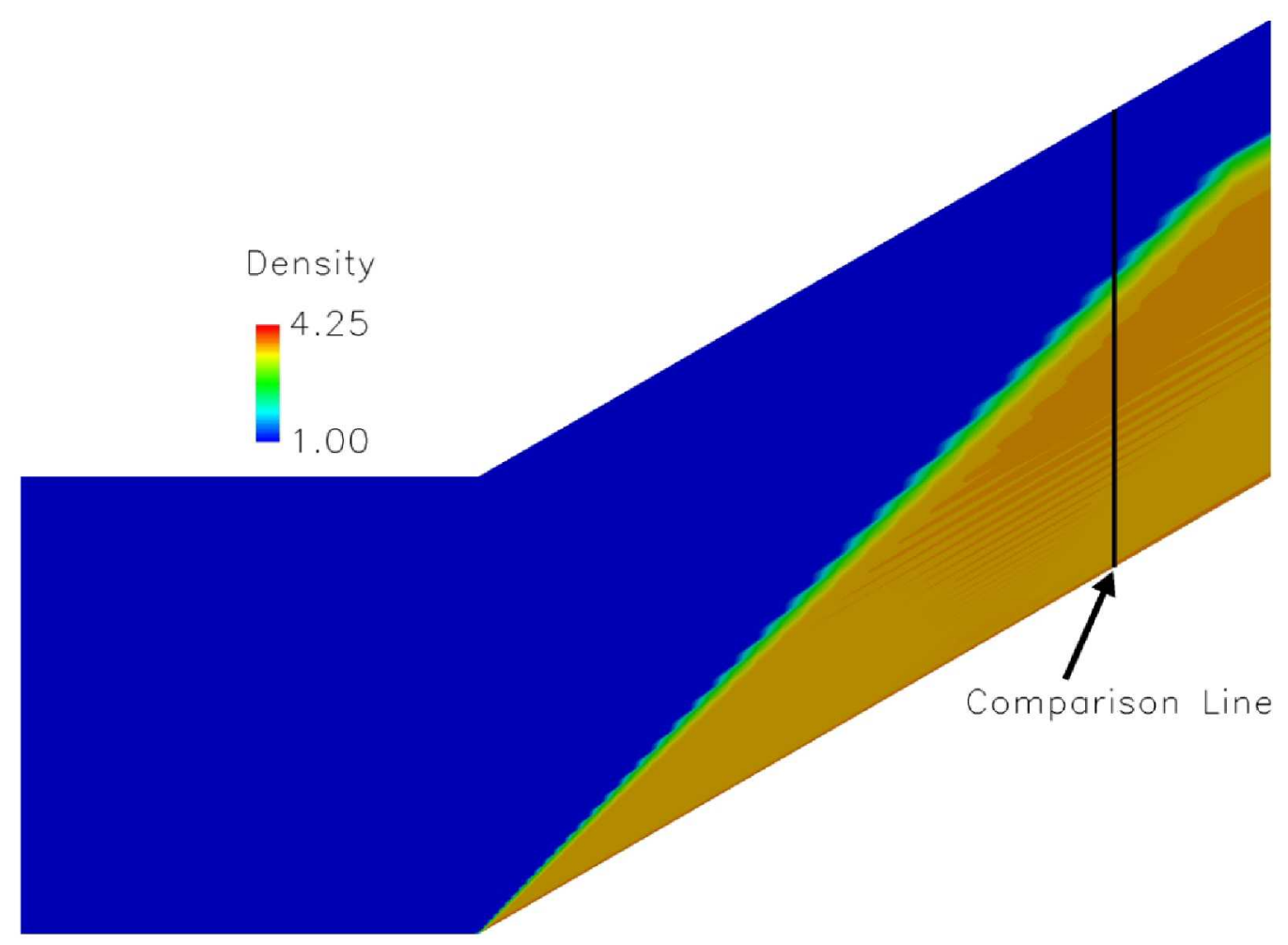

Figure 5c. Density contours using HLLE++ with $D E L T A=5$ for the $\mathrm{M}=4.030^{\circ} \mathrm{ramp}$. 

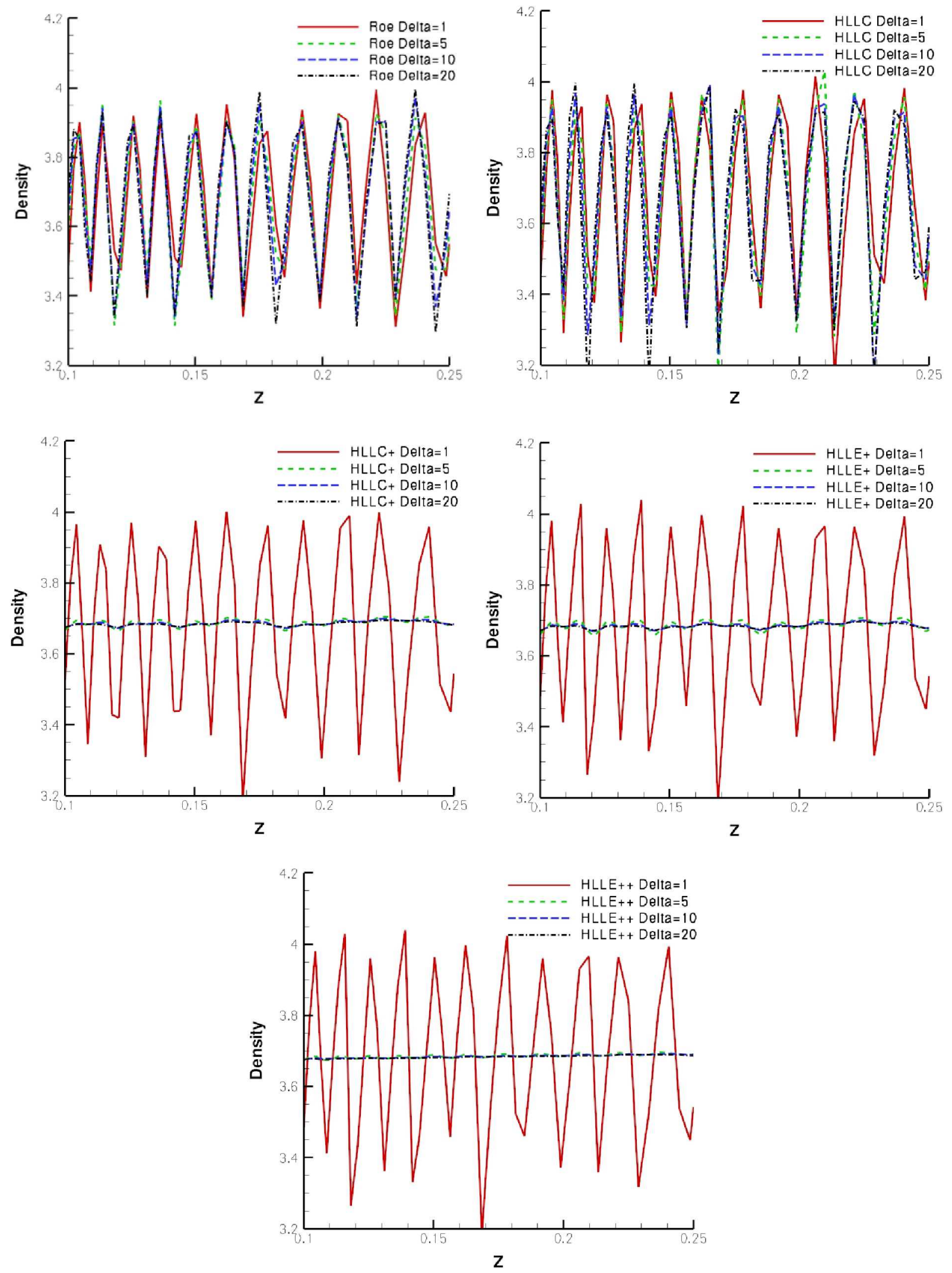

Figure 6. Density distribution on the comparison line for the $\mathrm{M}=4.030^{\circ}$ ramp. 


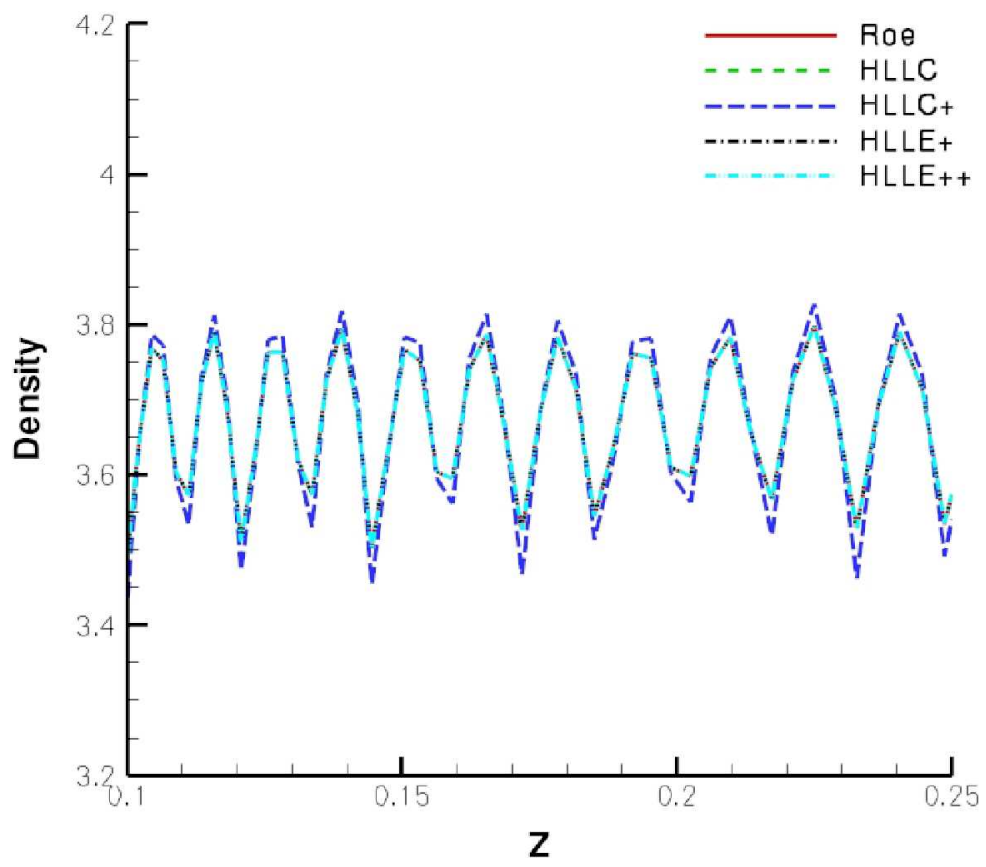

Figure 7. Density distribution on the comparison line using $1^{\text {st }}$ order spatial fluxes for the $M=4.030^{\circ}$ ramp.
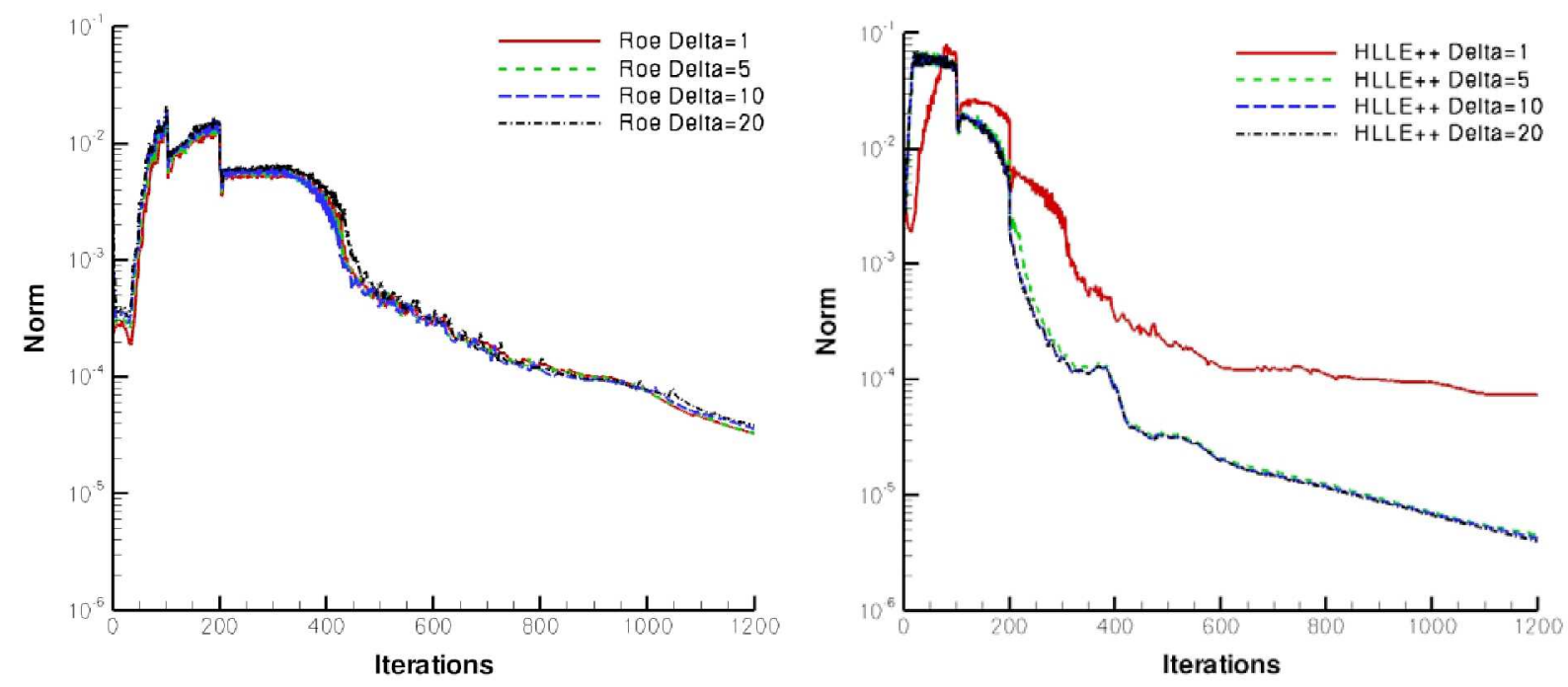

Figure 8. Convergence history for the $\mathrm{M}=4.030^{\circ}$ ramp. 


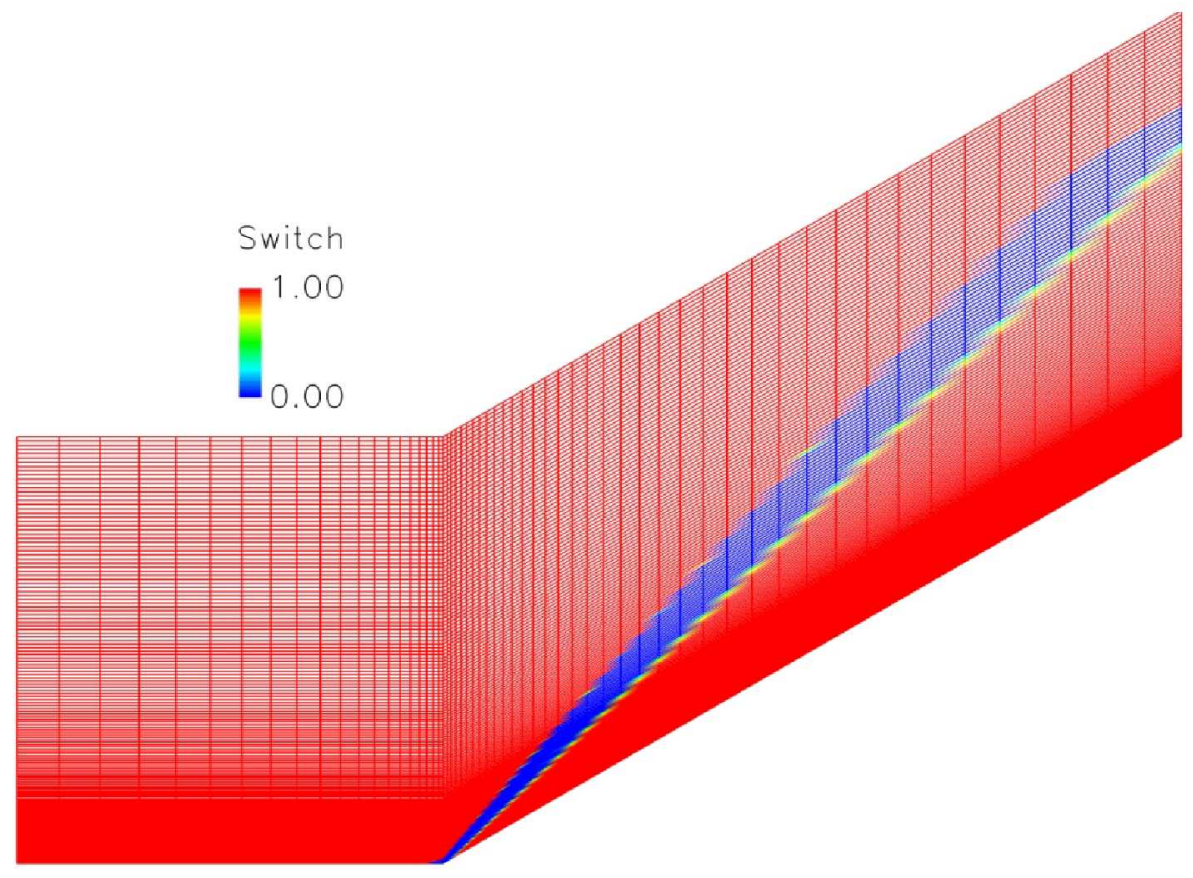

Figure 9. $\beta_{\text {New }}$ for the $\mathrm{M}=4.030^{\circ}$ ramp for $D E L T A=5$.

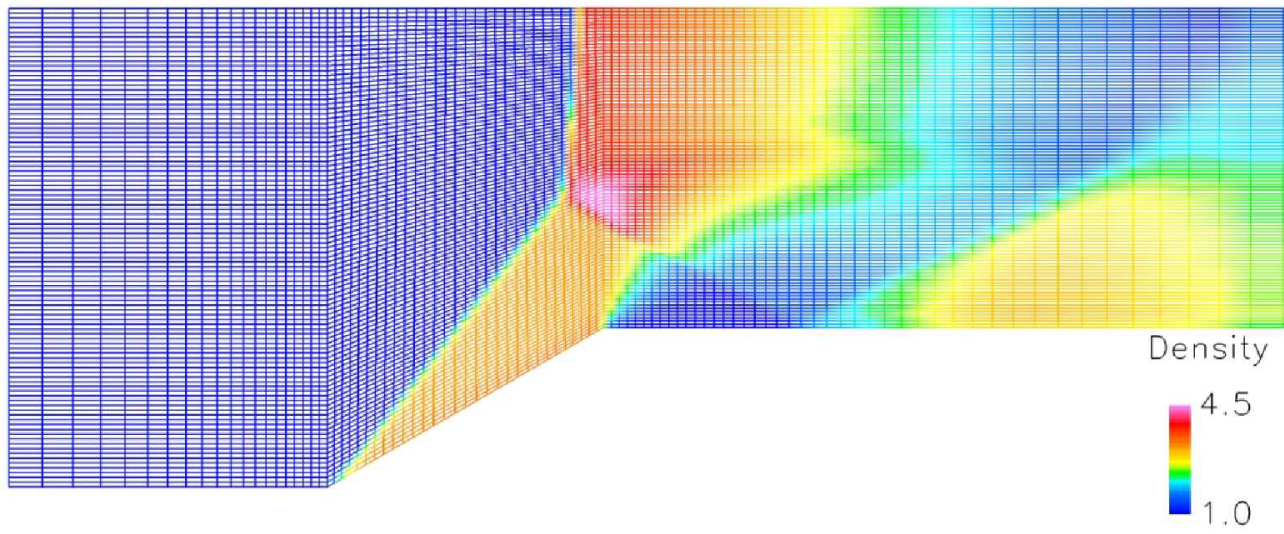

Figure 10. Grid colored by density for the $\mathrm{M}=3.030^{\circ} \mathrm{ramp}$ in a channel. 


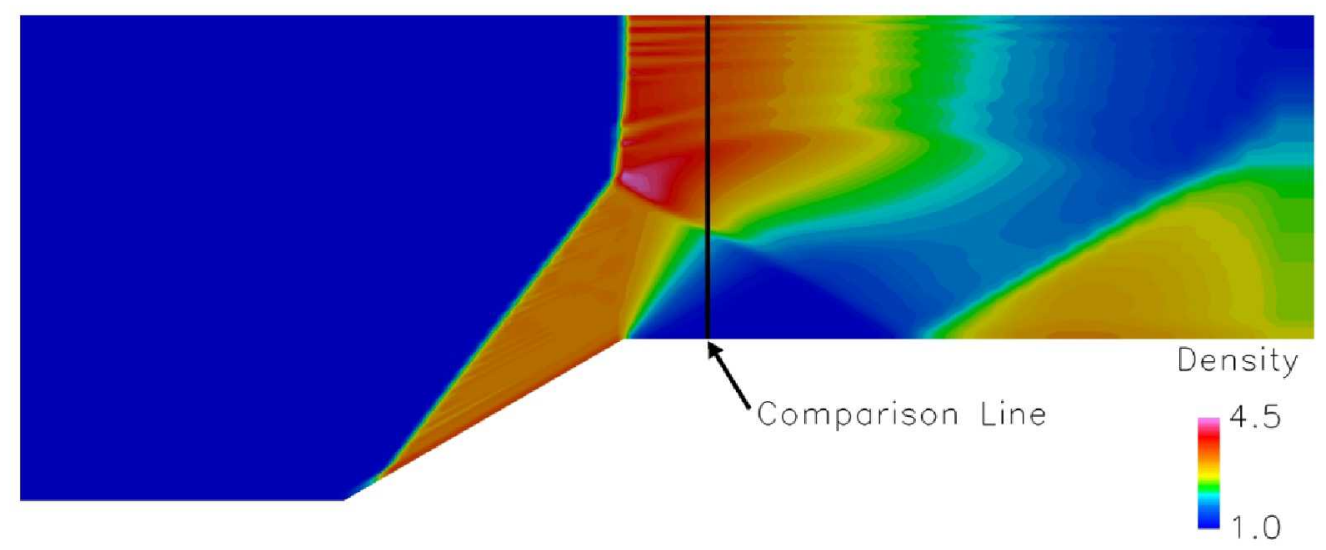

Figure 11a. Density contours using HLLC+ with $D E L T A=1$ for the $\mathrm{M}=3.030^{\circ}$ ramp in a channel.

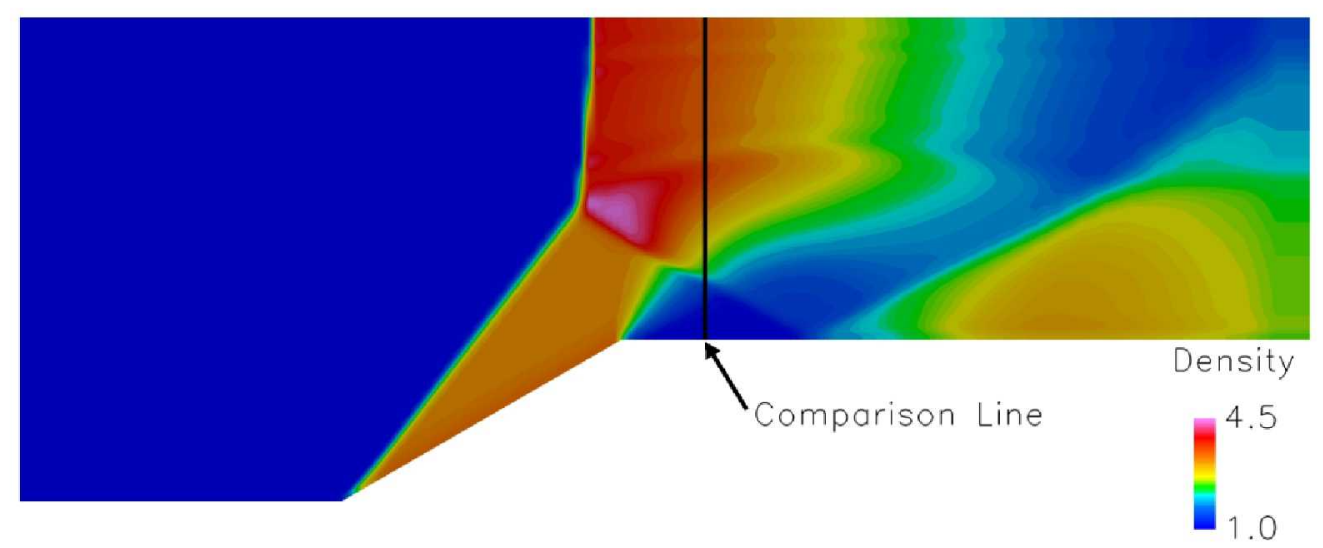

Figure 11b. Density contours using HLLC+ with $D E L T A=5$ for the $\mathrm{M}=3.030^{\circ}$ ramp in a channel.

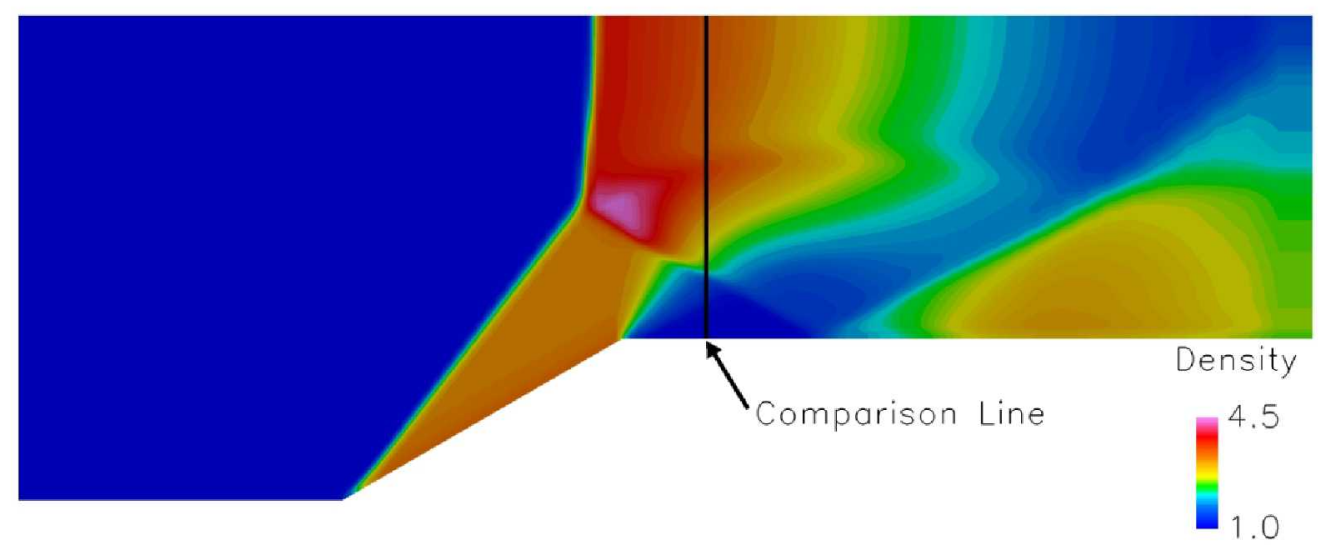

Figure 11c. Density contours using HLLE++ with $D E L T A=5$ for the $\mathrm{M}=3.030^{\circ} \mathrm{ramp}$ in a channel. 

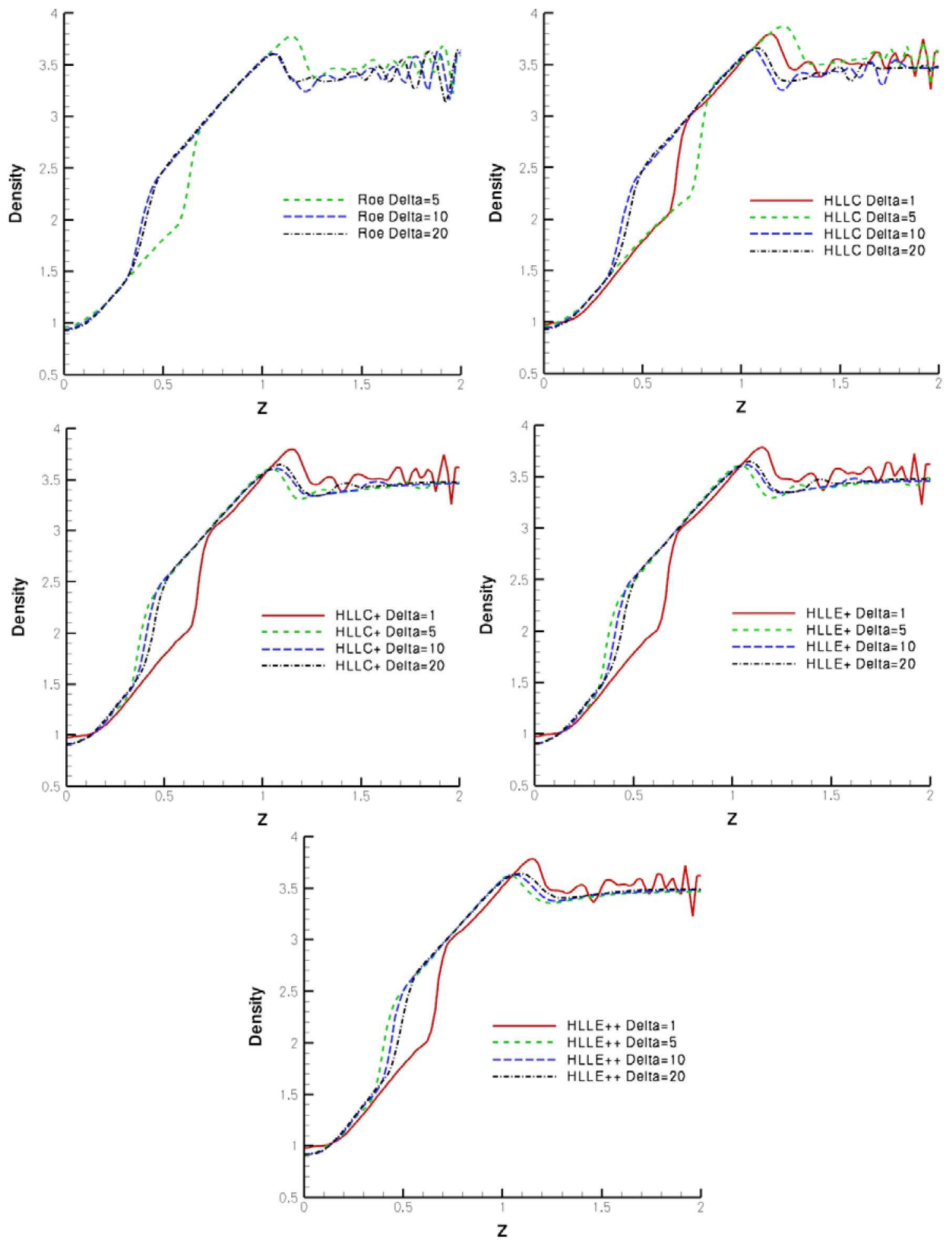

Figure 12. Density distribution on the comparison line for the $\mathrm{M}=3.030^{\circ}$ ramp in a channel. 

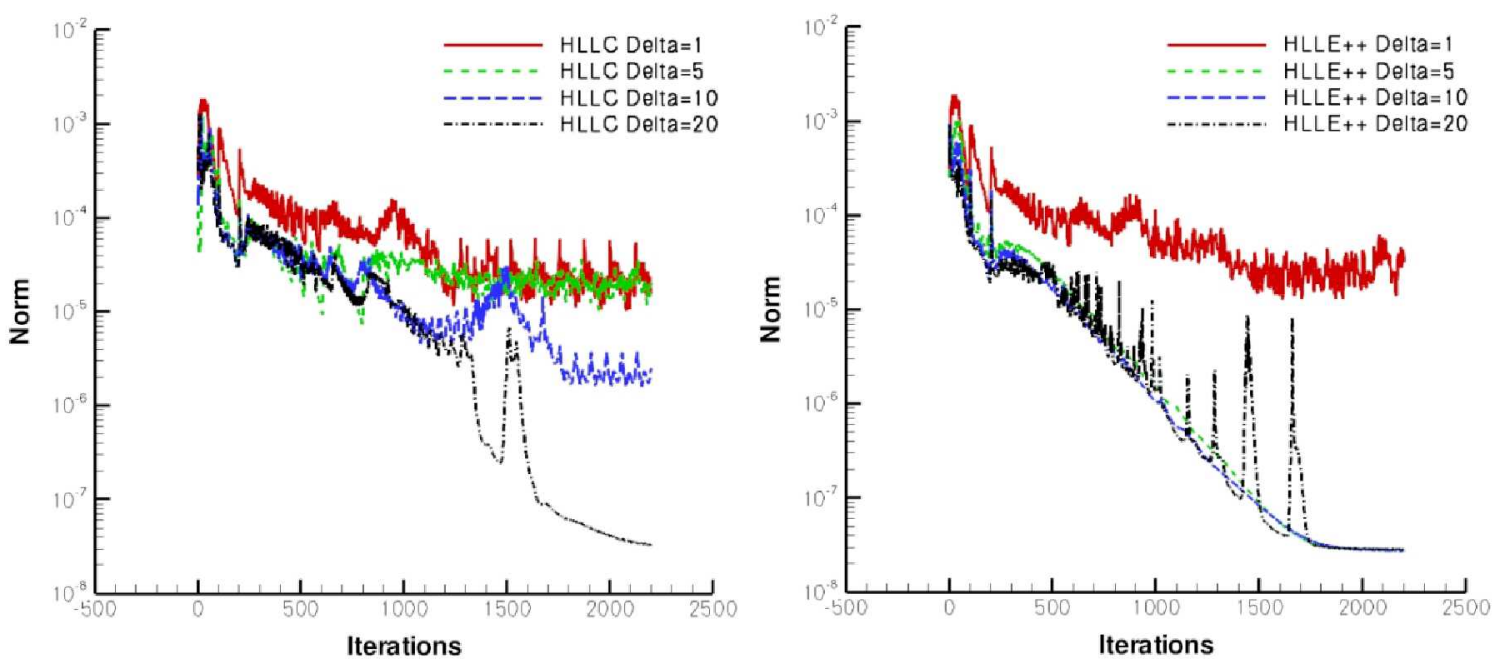

Figure 13. Convergence history for the $\mathrm{M}=3.030^{\circ}$ ramp in a channel.

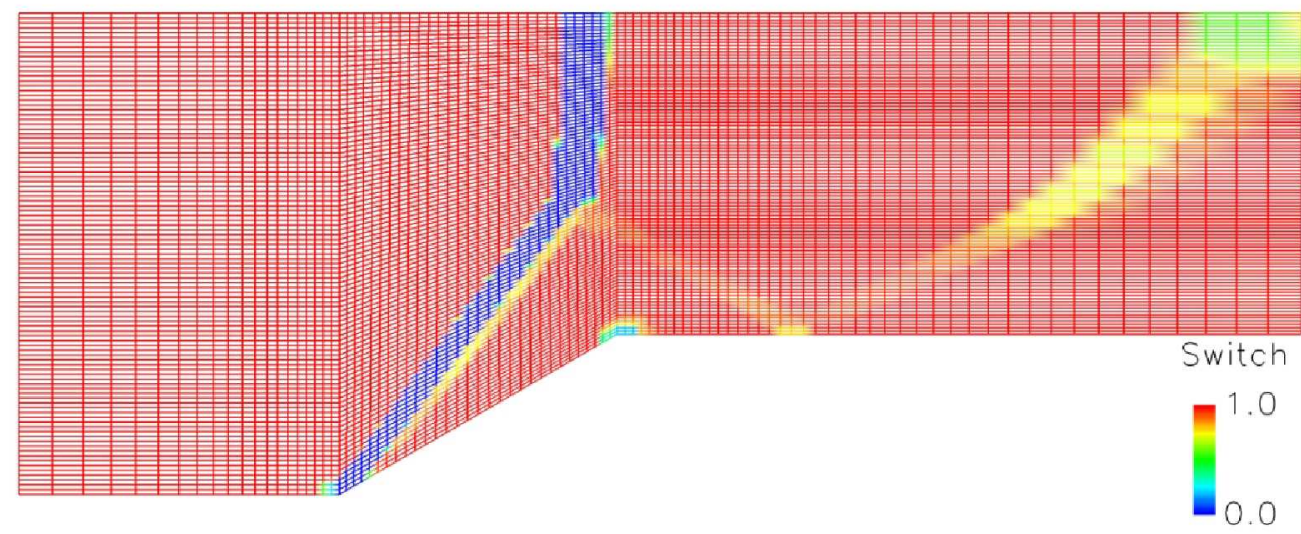

Figure 14. $\beta_{\text {New }}$ for the $\mathrm{M}=3.030^{\circ}$ ramp in a channel for $D E L T A=5$.
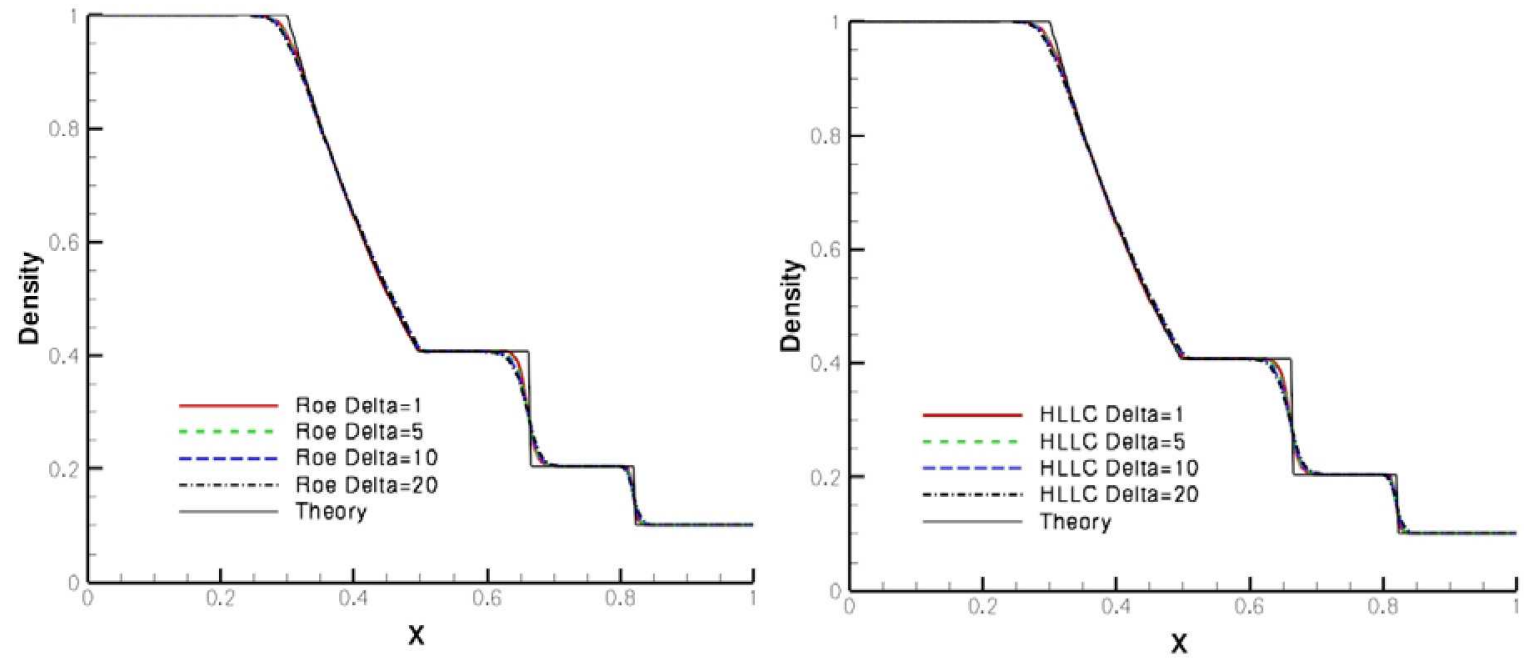

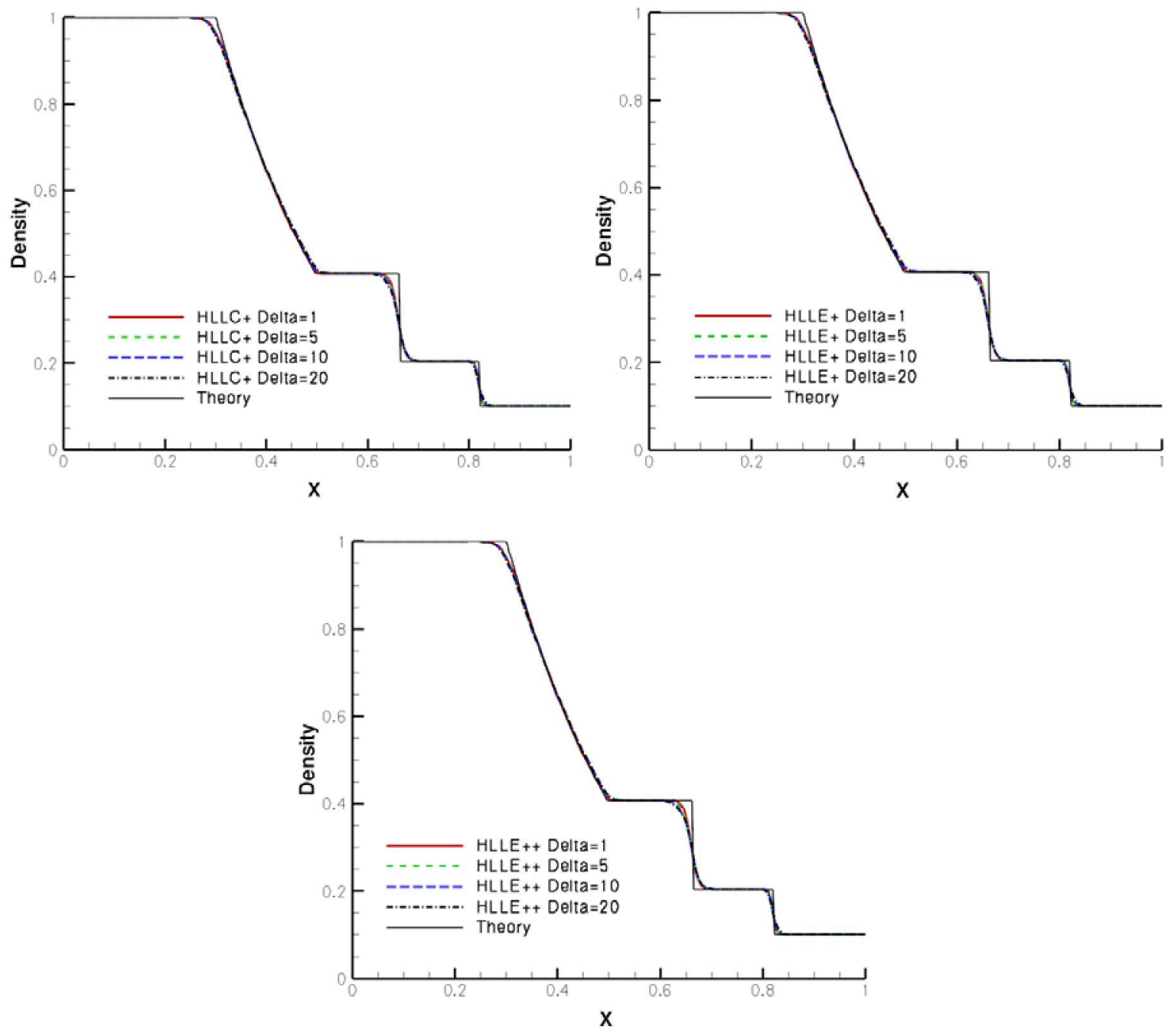

Figure 15. Density distribution in a shock tube.

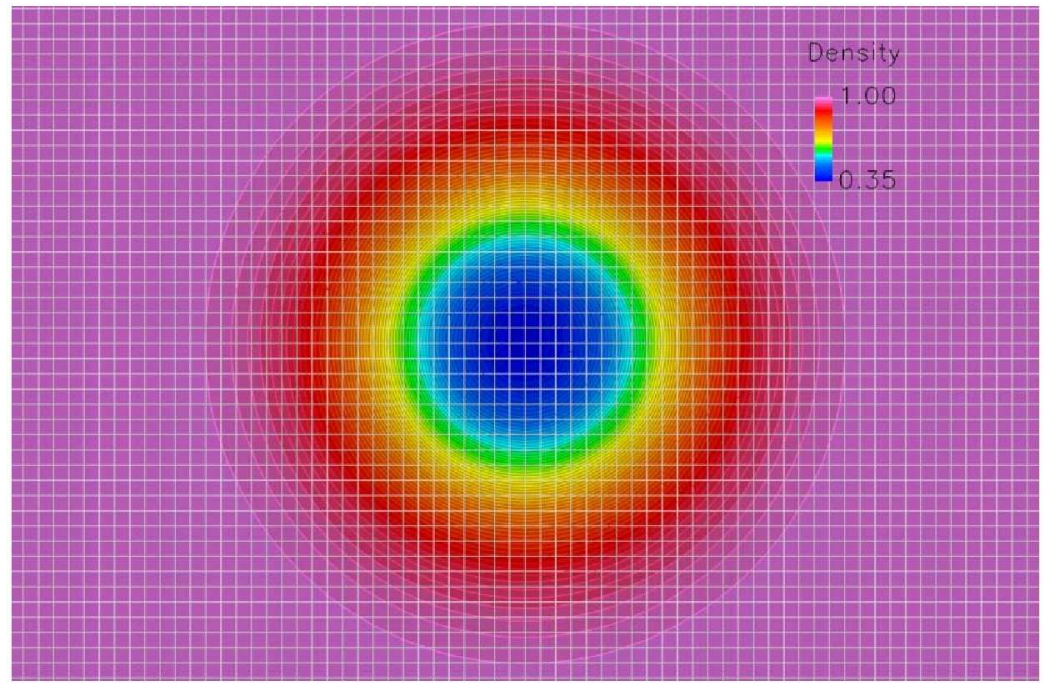

Figure 16. Initial density contours for the convecting vortex. 


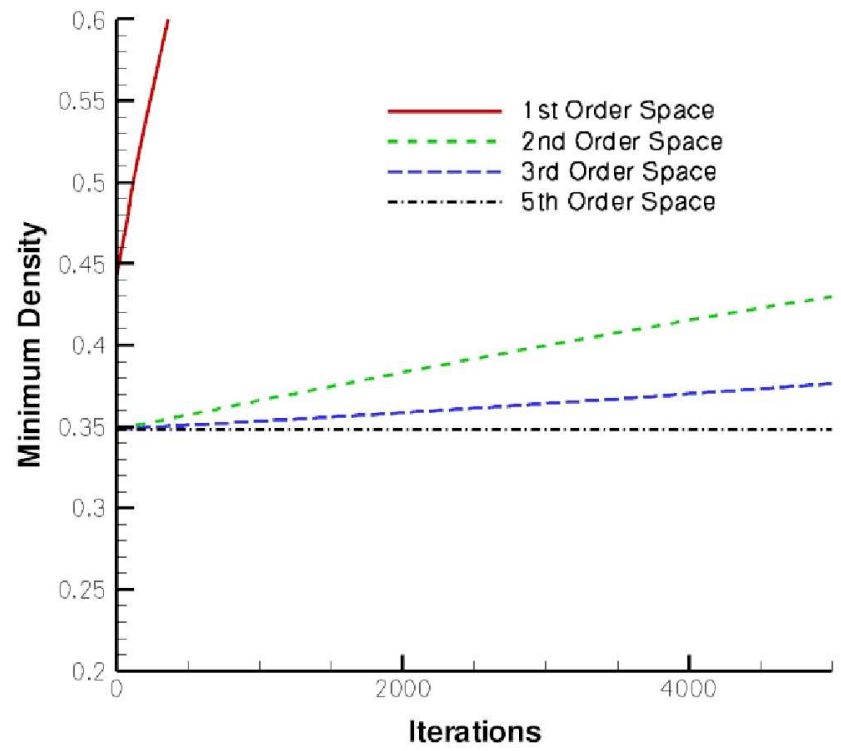

Figure 17. Effect of spatial order for the inviscid flux terms for the convecting vortex.
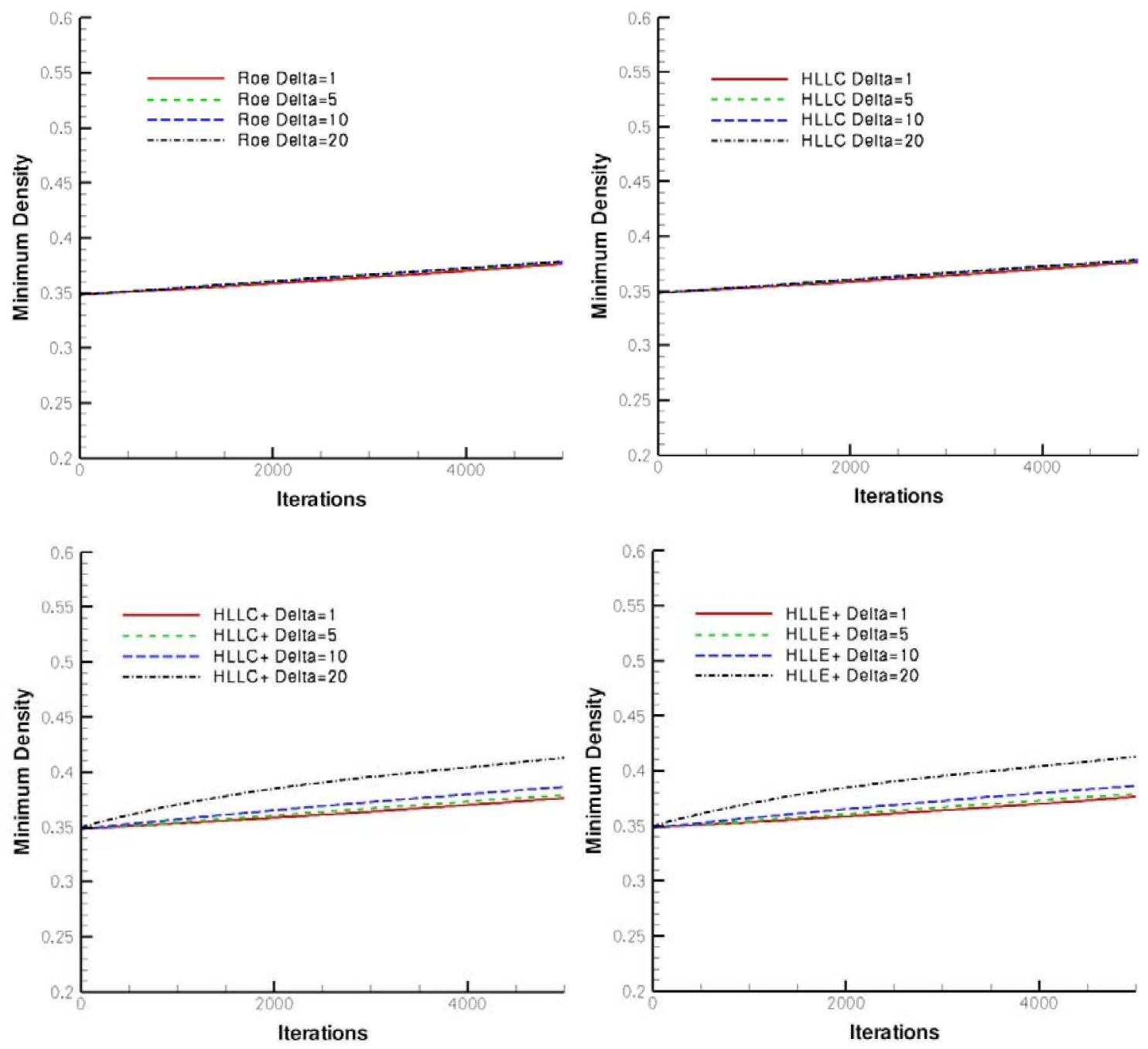


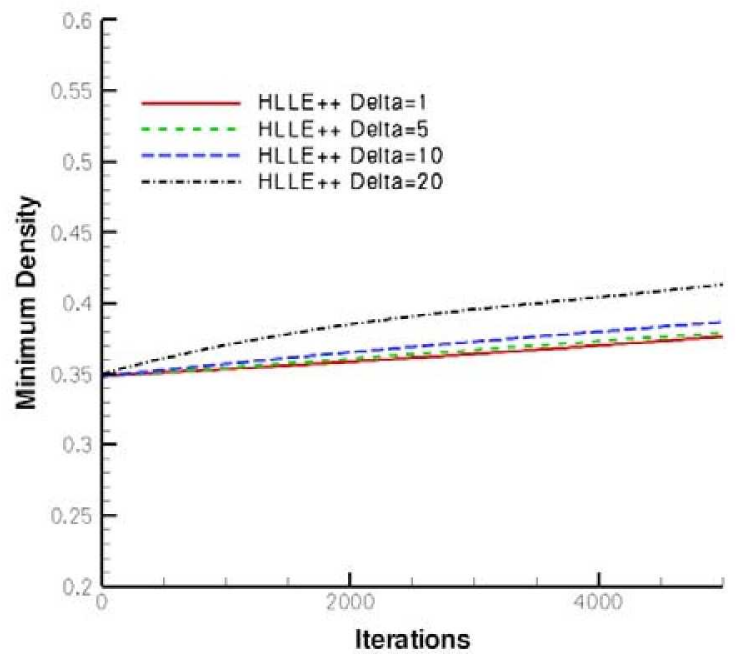

Figure 18. Minimum density for the convecting vortex.
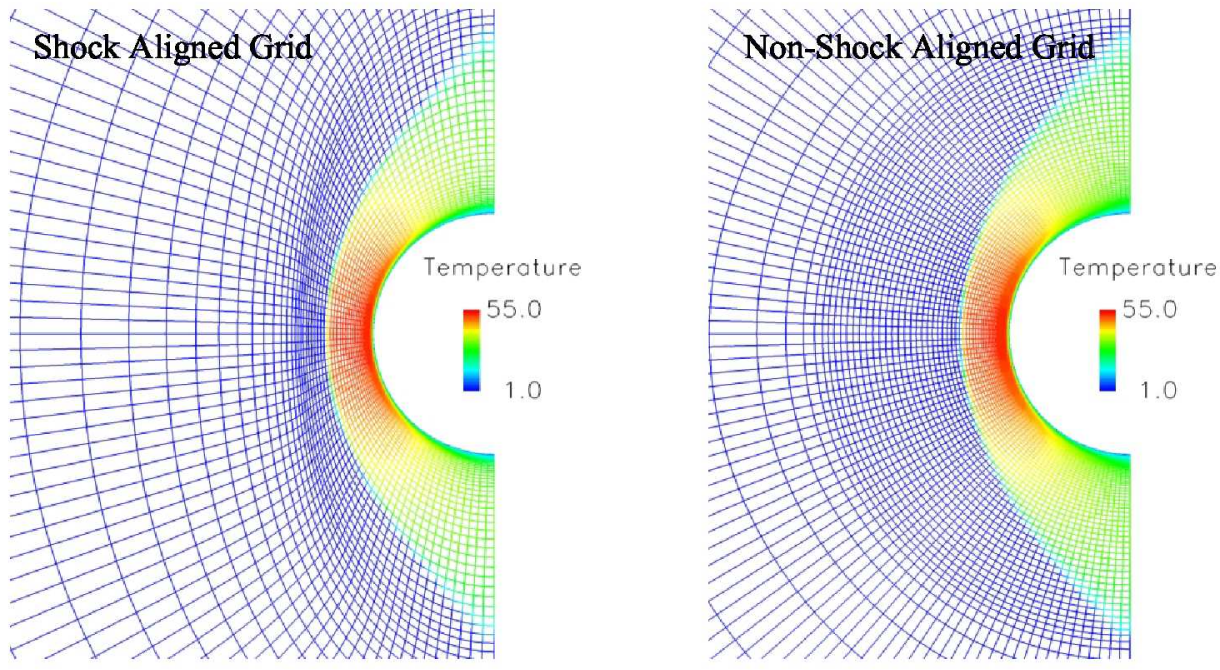

Figure 19. Grids colored by temperature. 

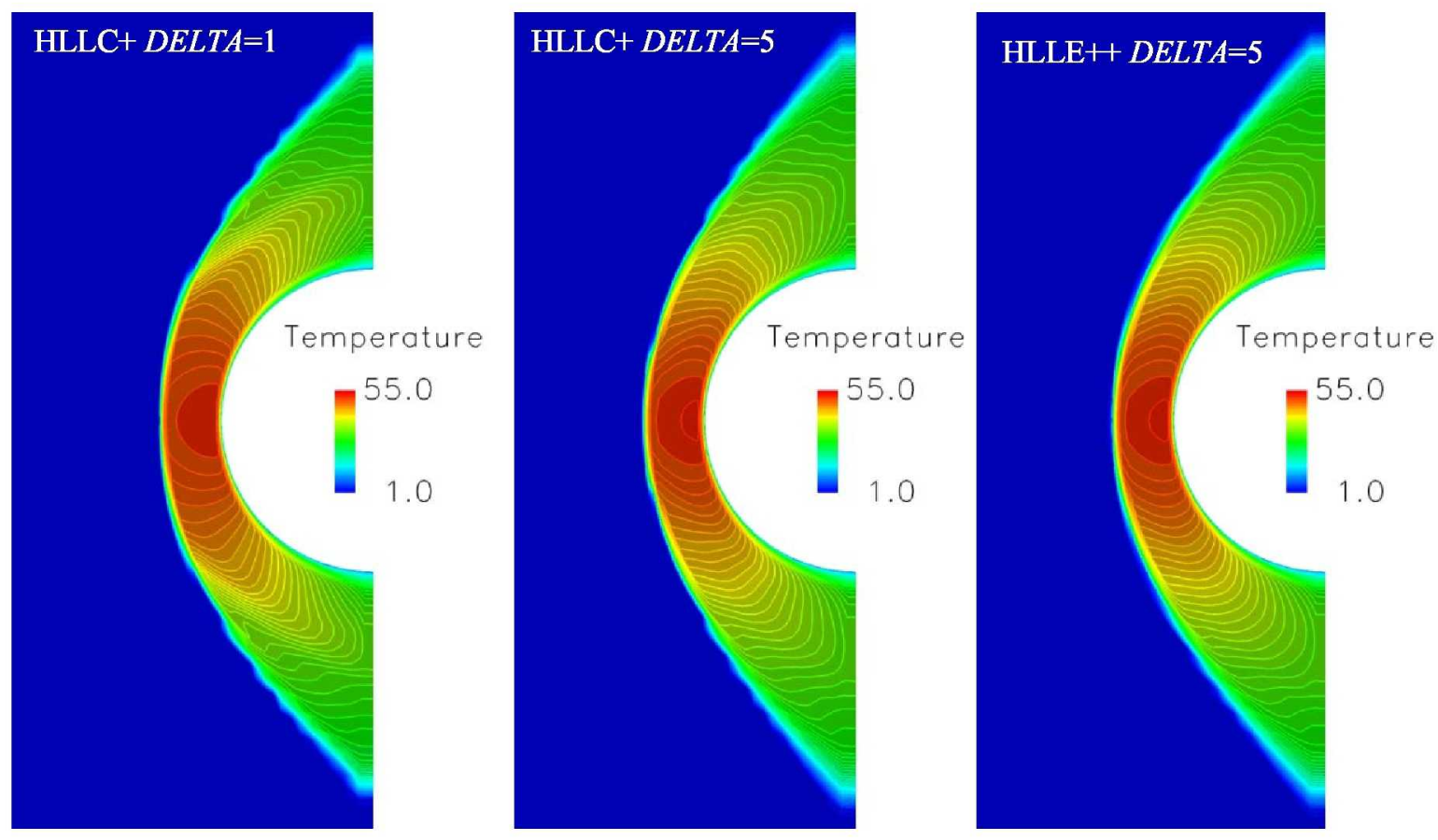

Figure 20. Temperature distribution for the $\mathrm{M}=16.01$ cylinder bow shock.
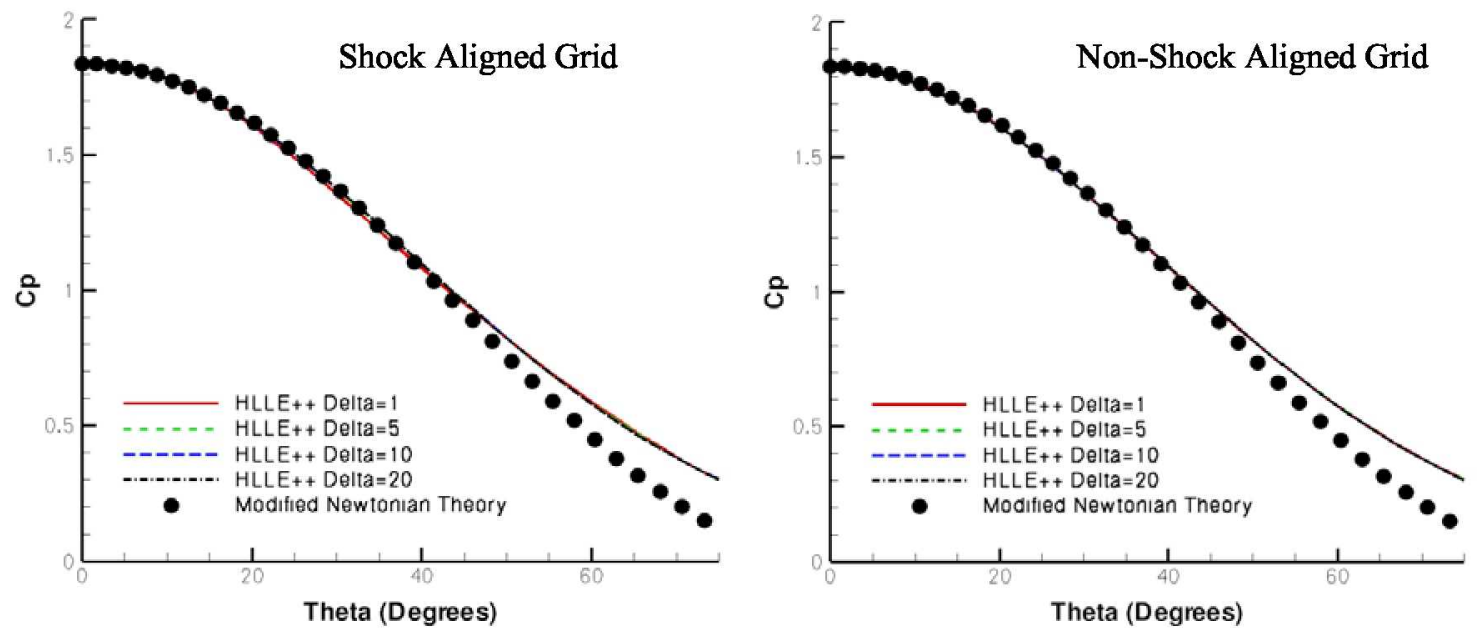

Figure 21. Pressure coefficient for the $\mathrm{M}=16.01$ cylinder bow shock. 

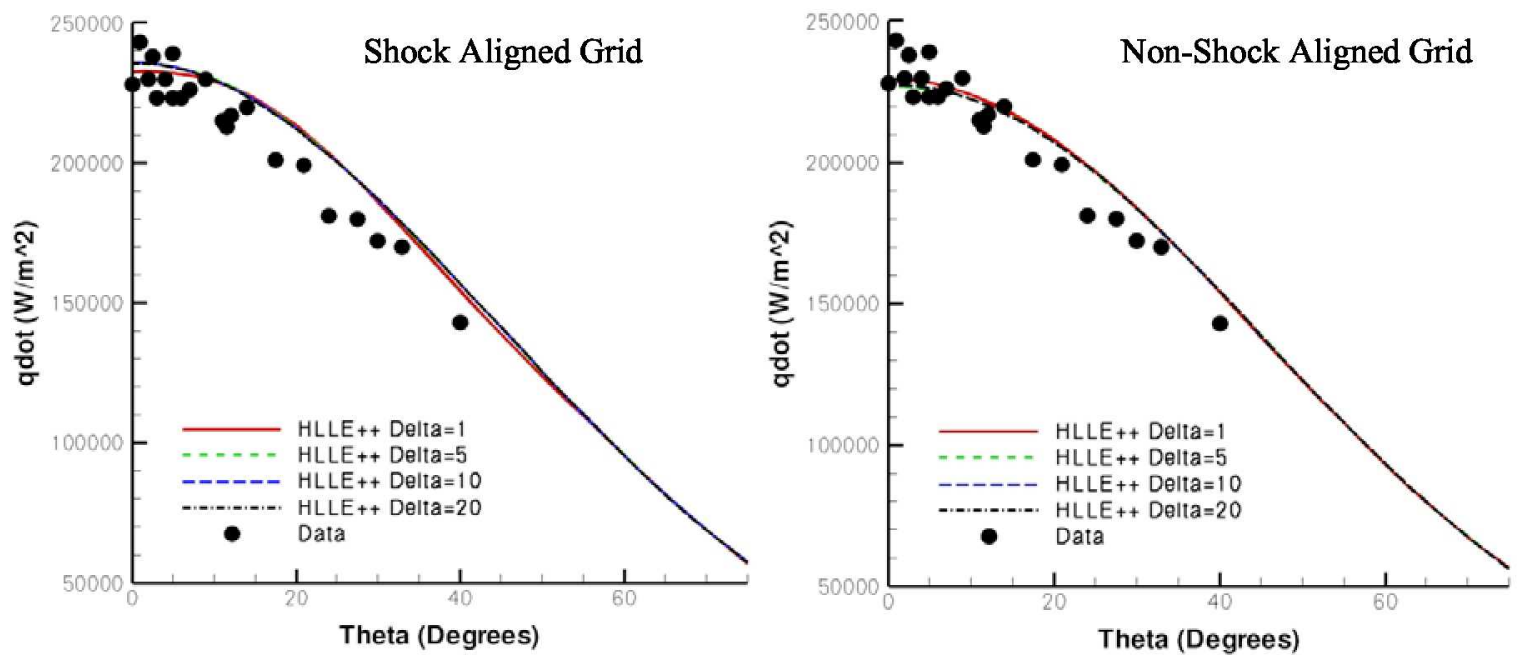

Figure 22. Heat transfer for the $M=16.01$ cylinder bow shock.
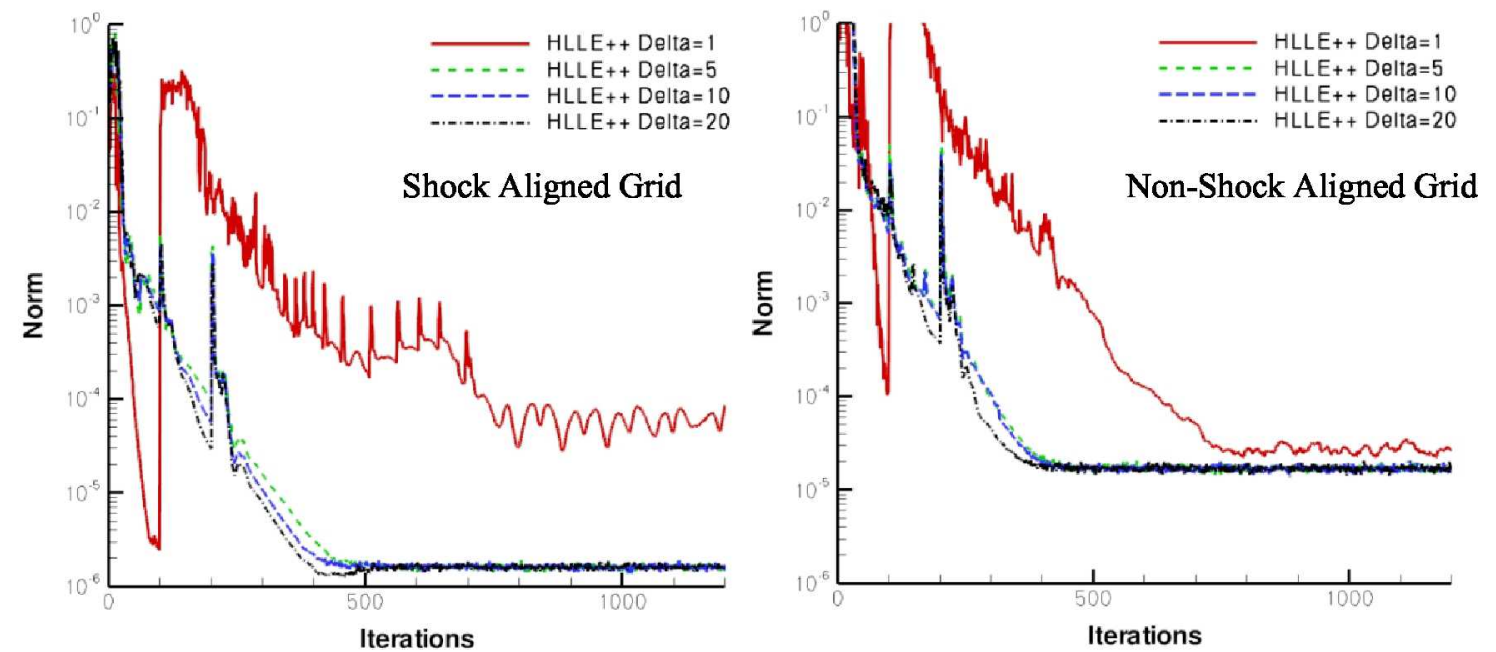

Figure 23. Convergence history for the $\mathrm{M}=16.01$ cylinder bow shock. 


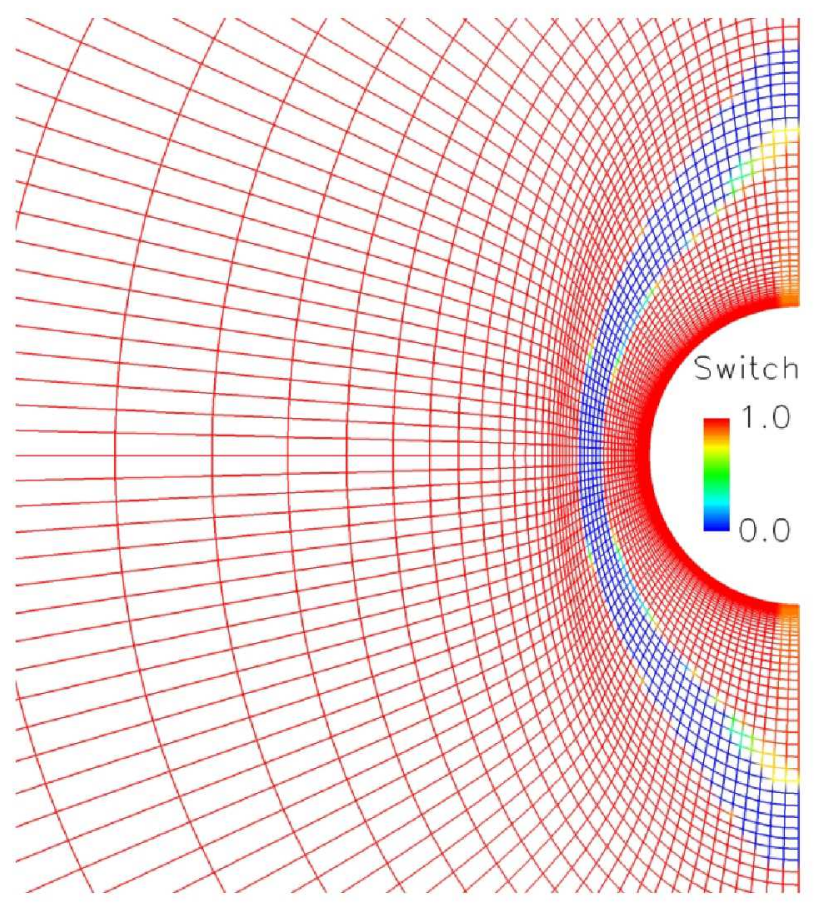

Figure 24. $\beta_{\text {New }}$ for the $\mathrm{M}=16.01$ cylinder bow shock for $D E L T A=5$.

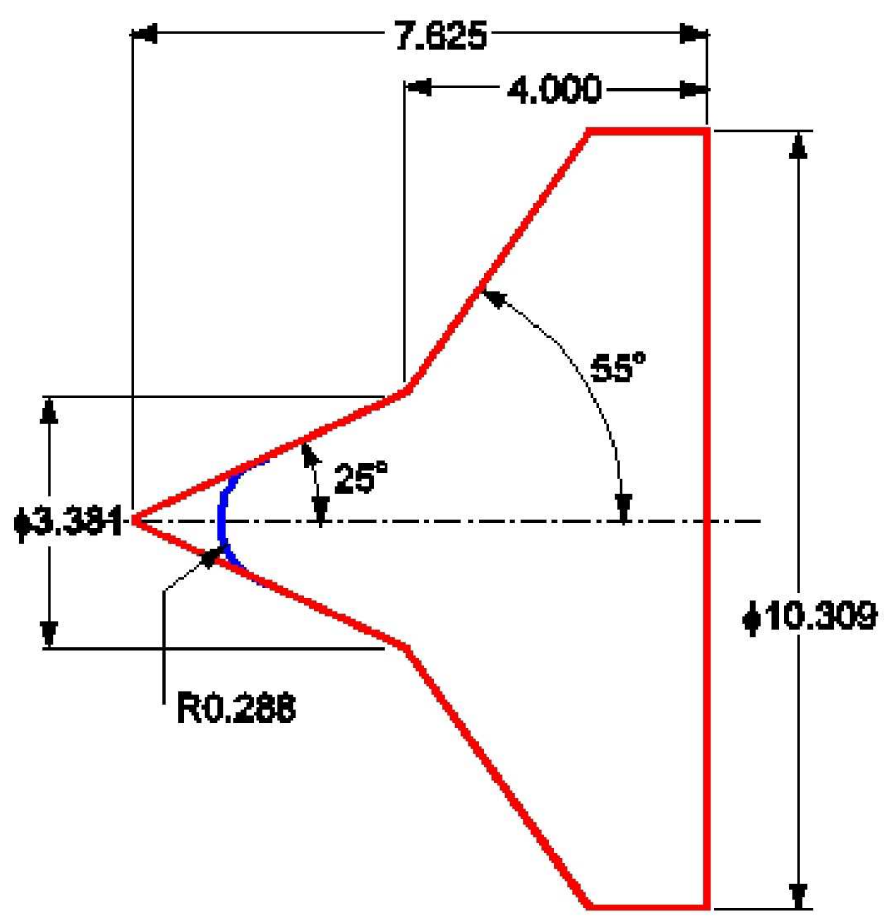

Figure 25. Geometry for the hypersonic double cone (all dimensions in inches). 


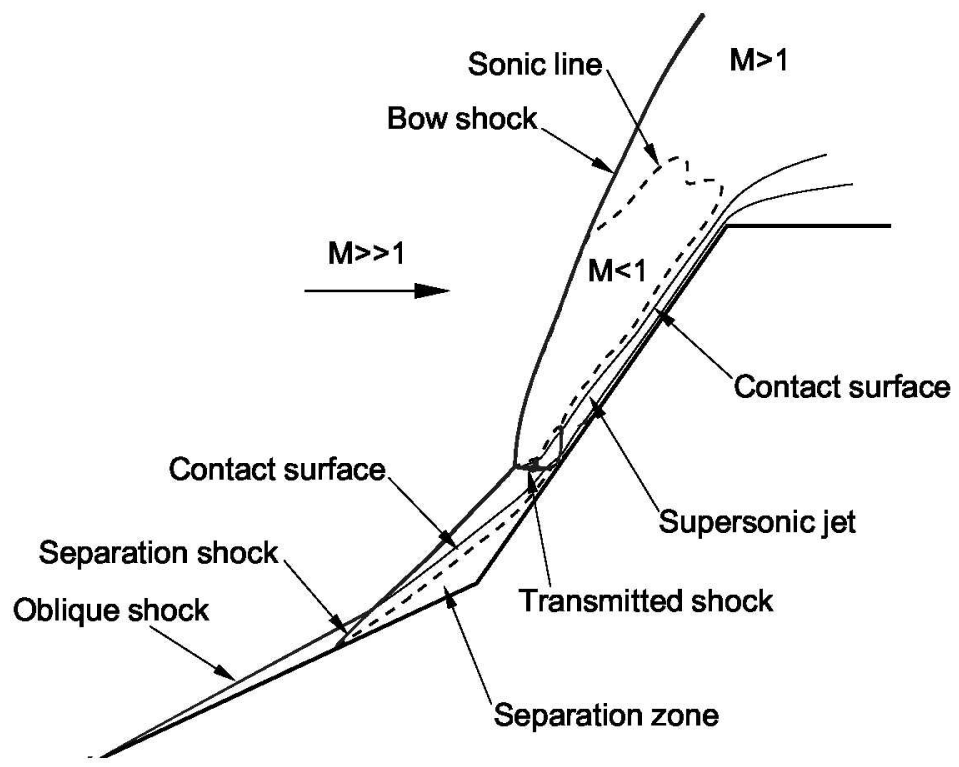

Figure 26. Flow field features for the double cone experiment.

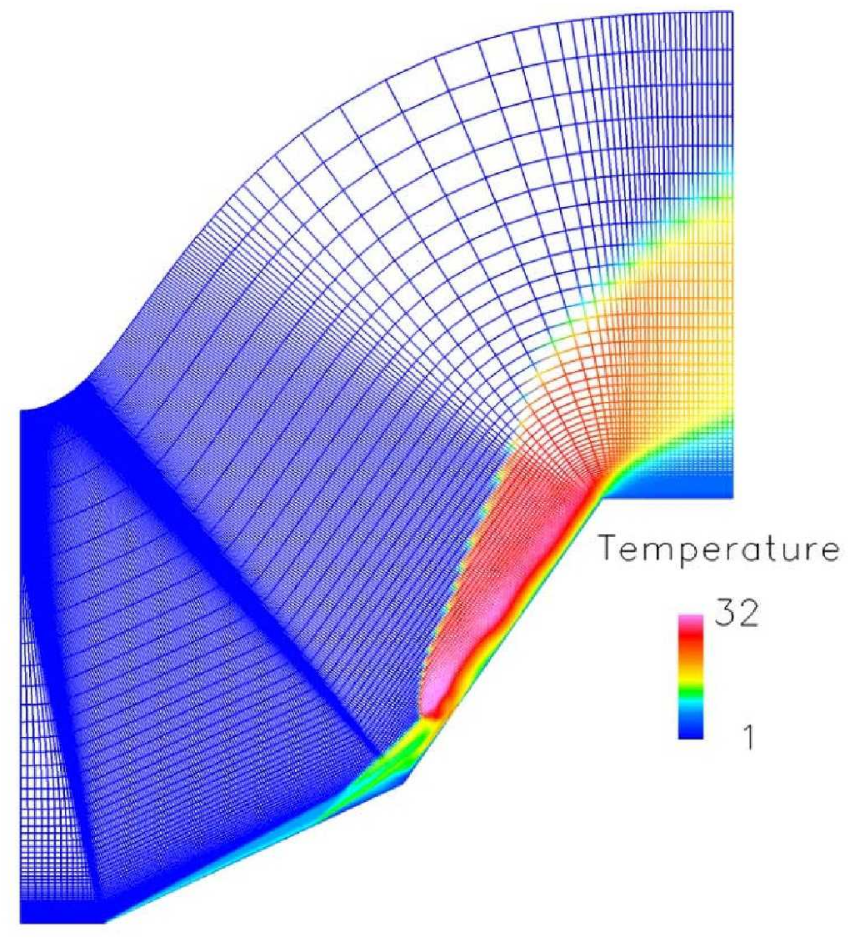

Figure 27. Grid colored by temperature for the hypersonic double cone. 

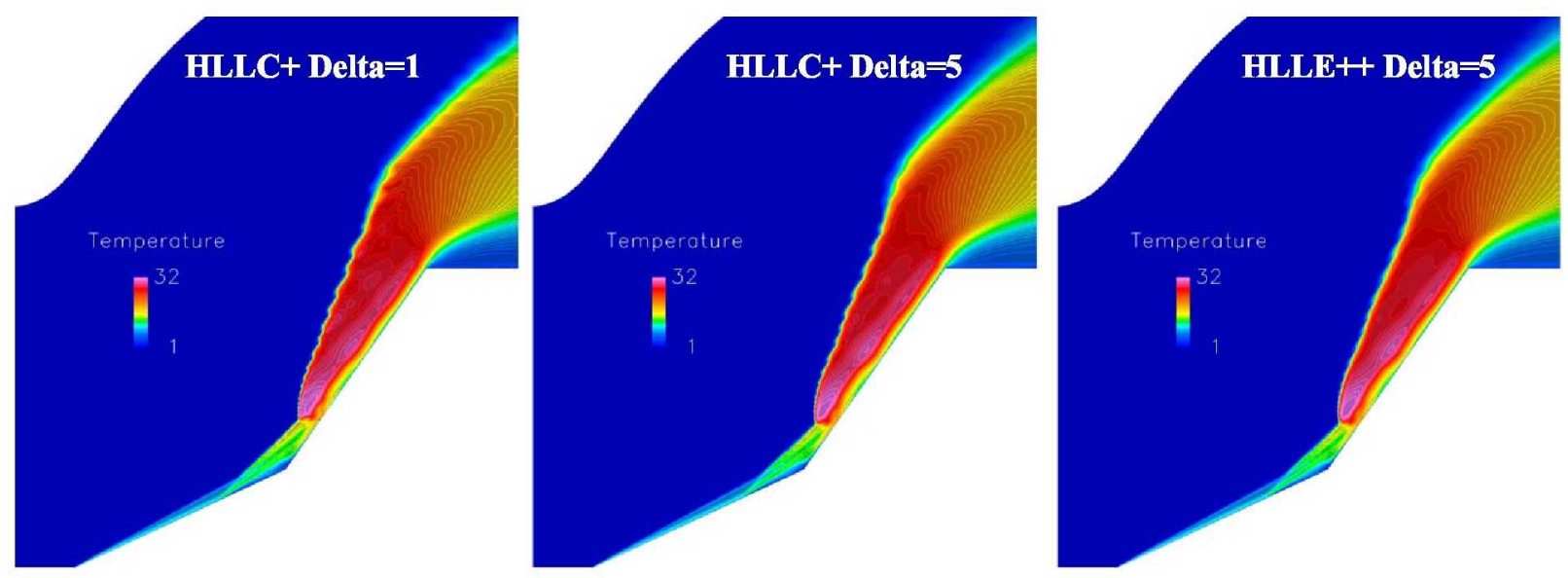

Figure 28. Temperature contours for the hypersonic double cone.
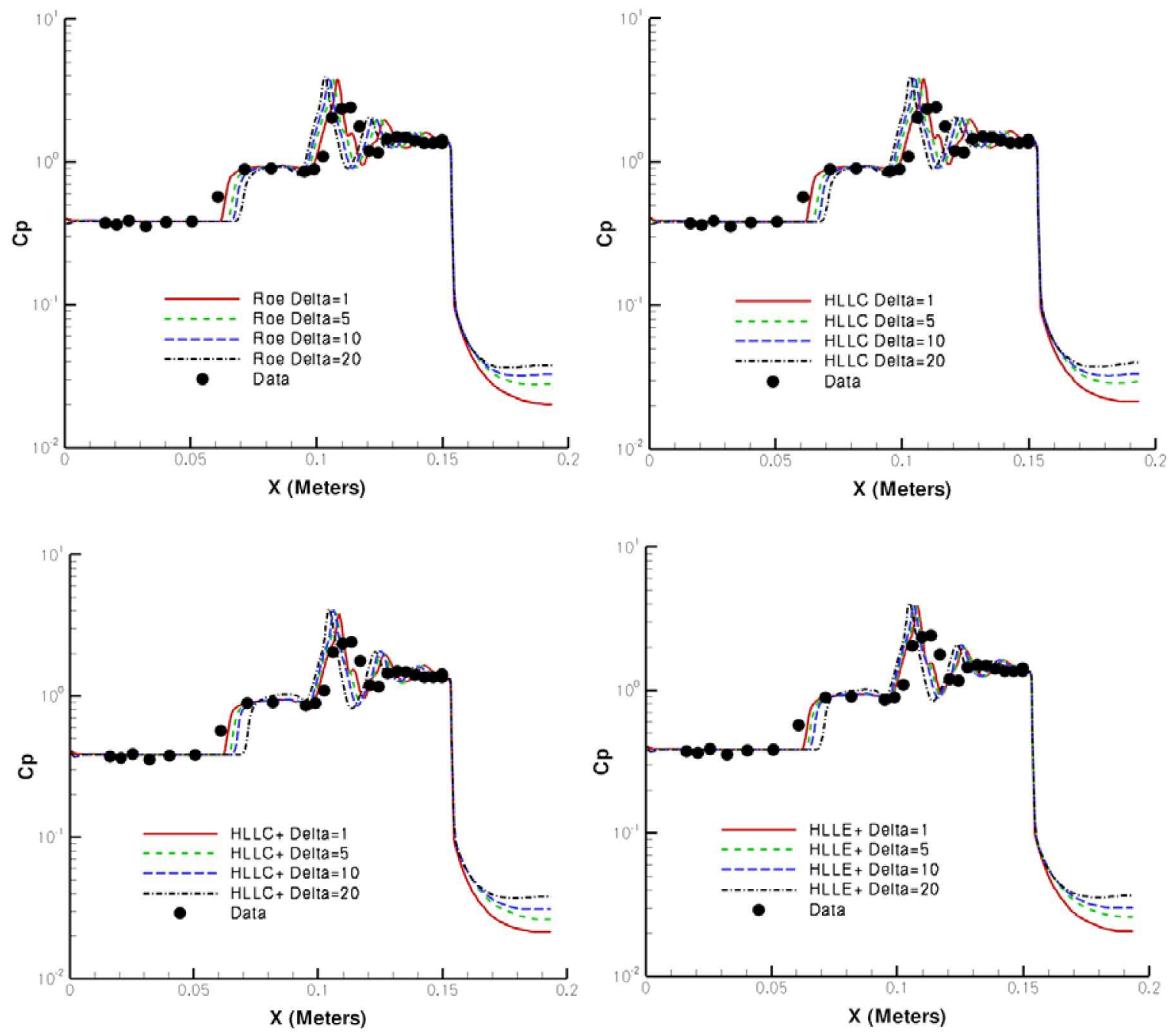


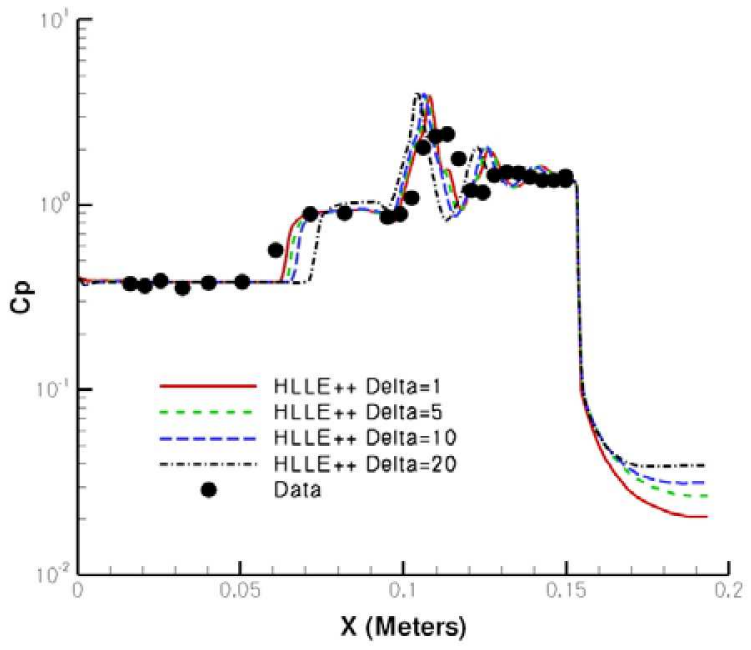

Figure 29. Pressure coefficient for the hypersonic double cone.
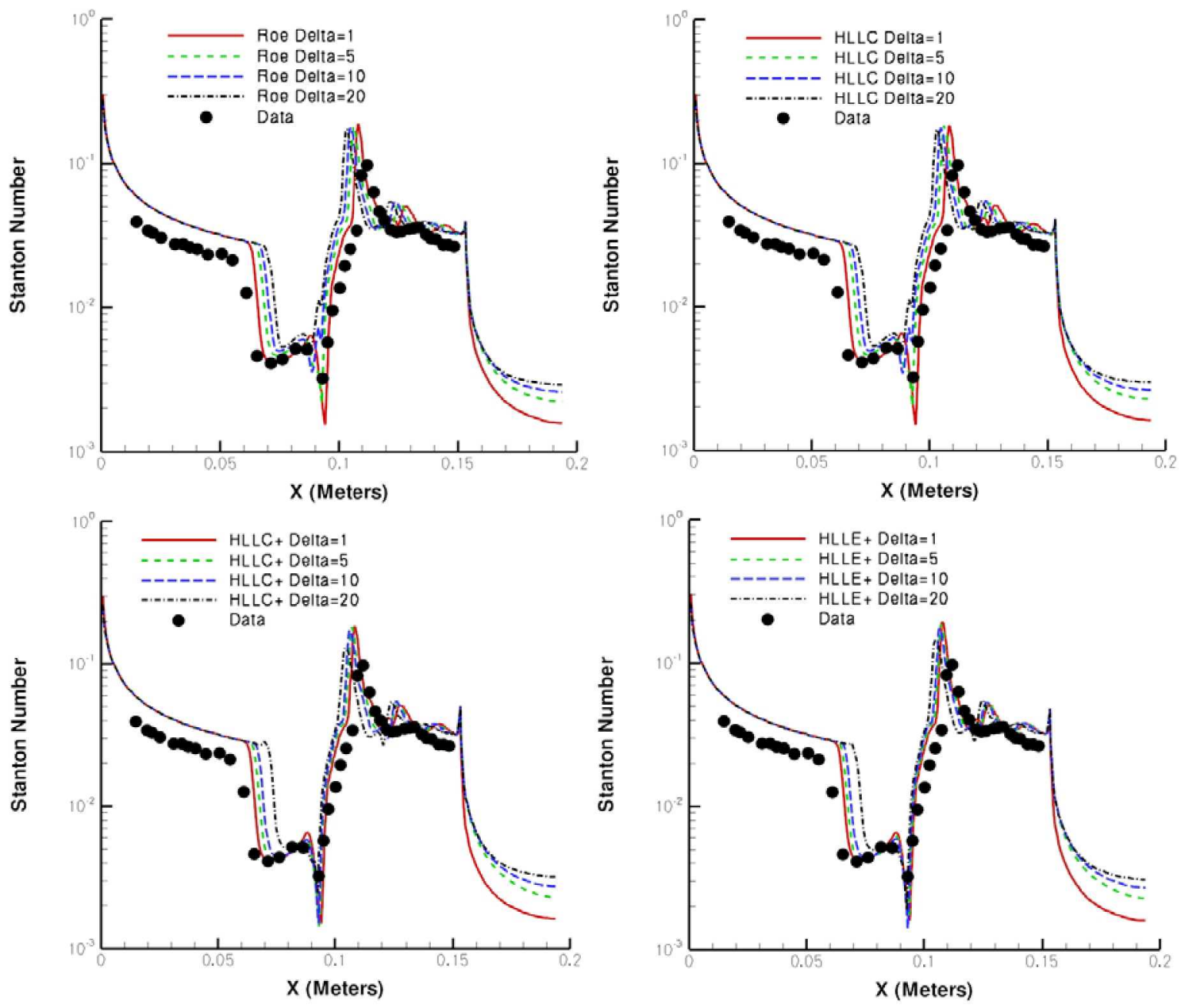


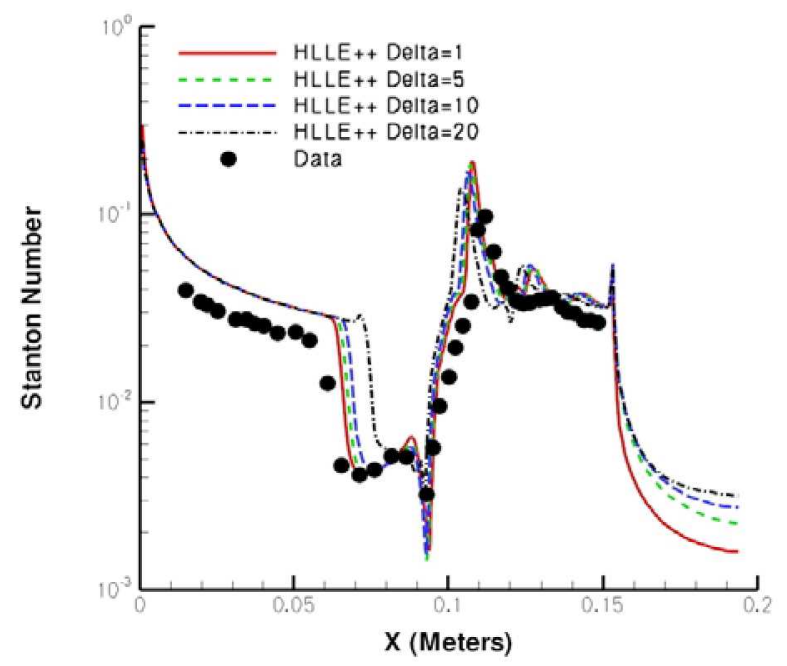

Figure 30. Heat transfer for the hypersonic double cone.

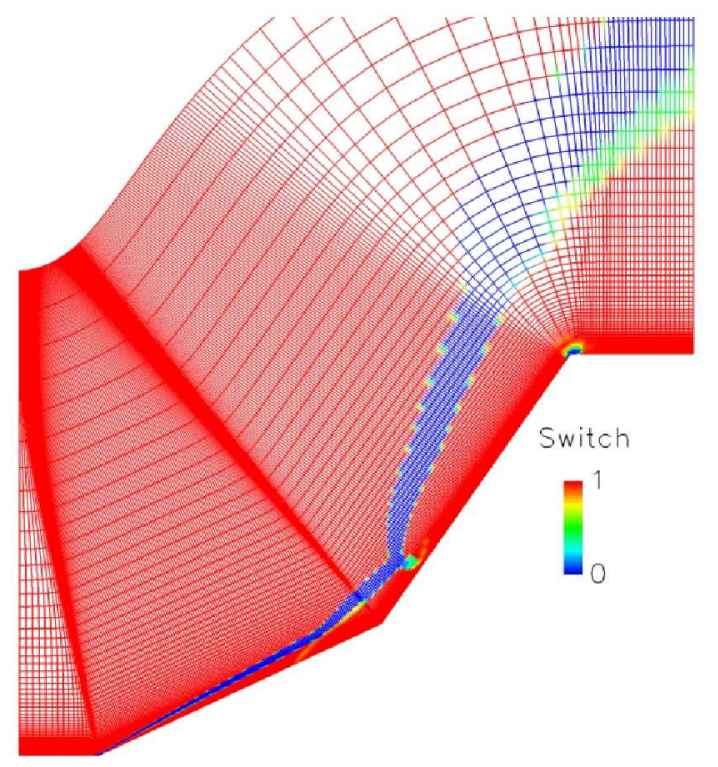

Figure 31. $\beta_{N e w}$ for the hypersonic double cone for $D E L T A=5$. 

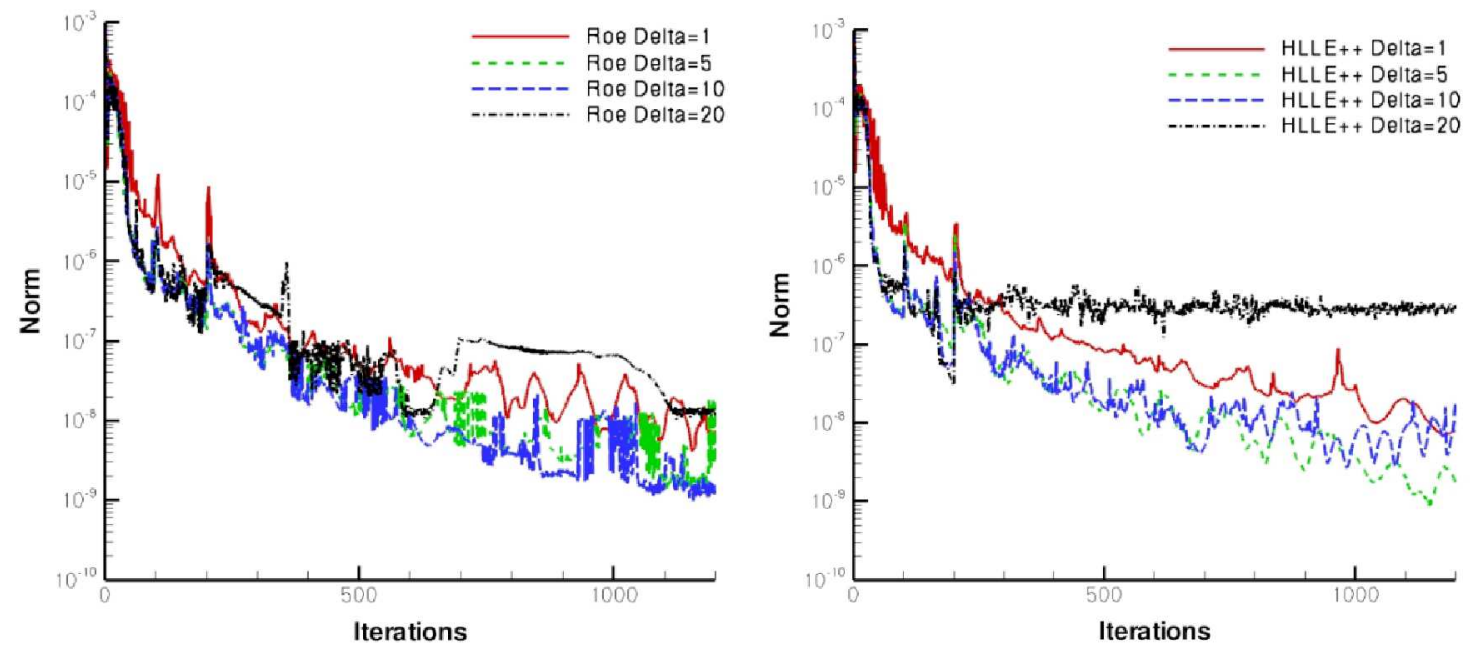

Figure 32. Convergence history for the hypersonic double cone.

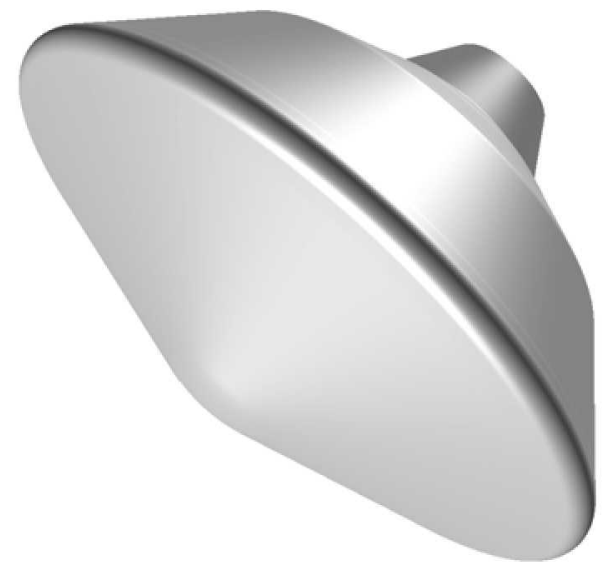

Figure 33. Capsule geometry.
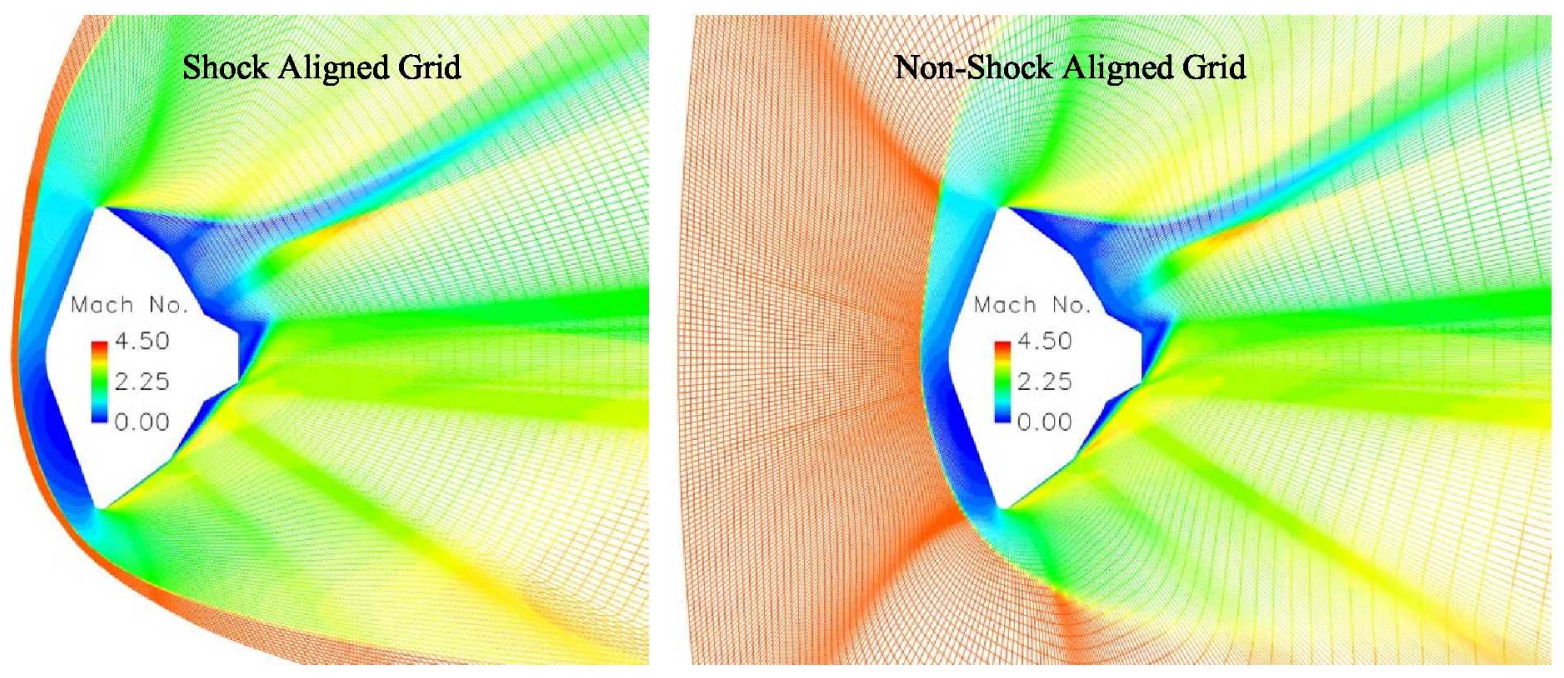

Figure 34. Grid on symmetry plane for capsule. 

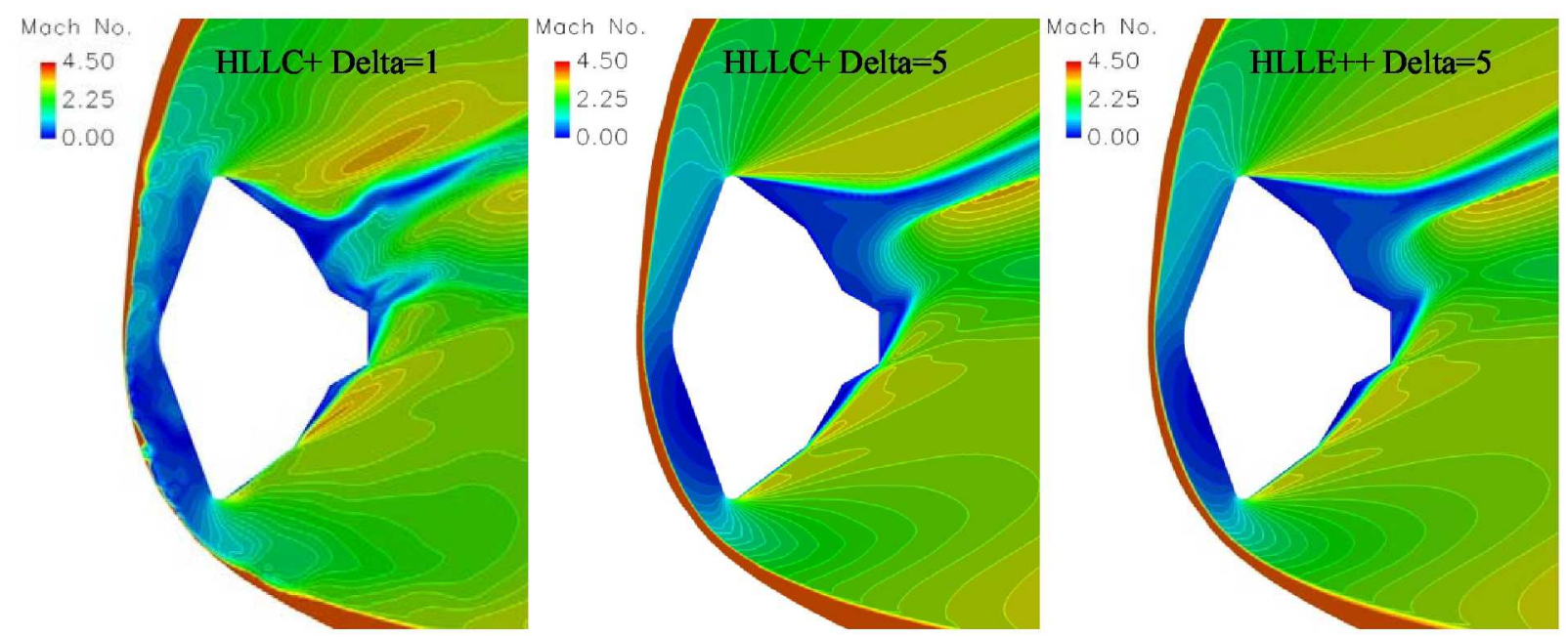

Figure 35. Mach number on the symmetry plane for the shock aligned grid.
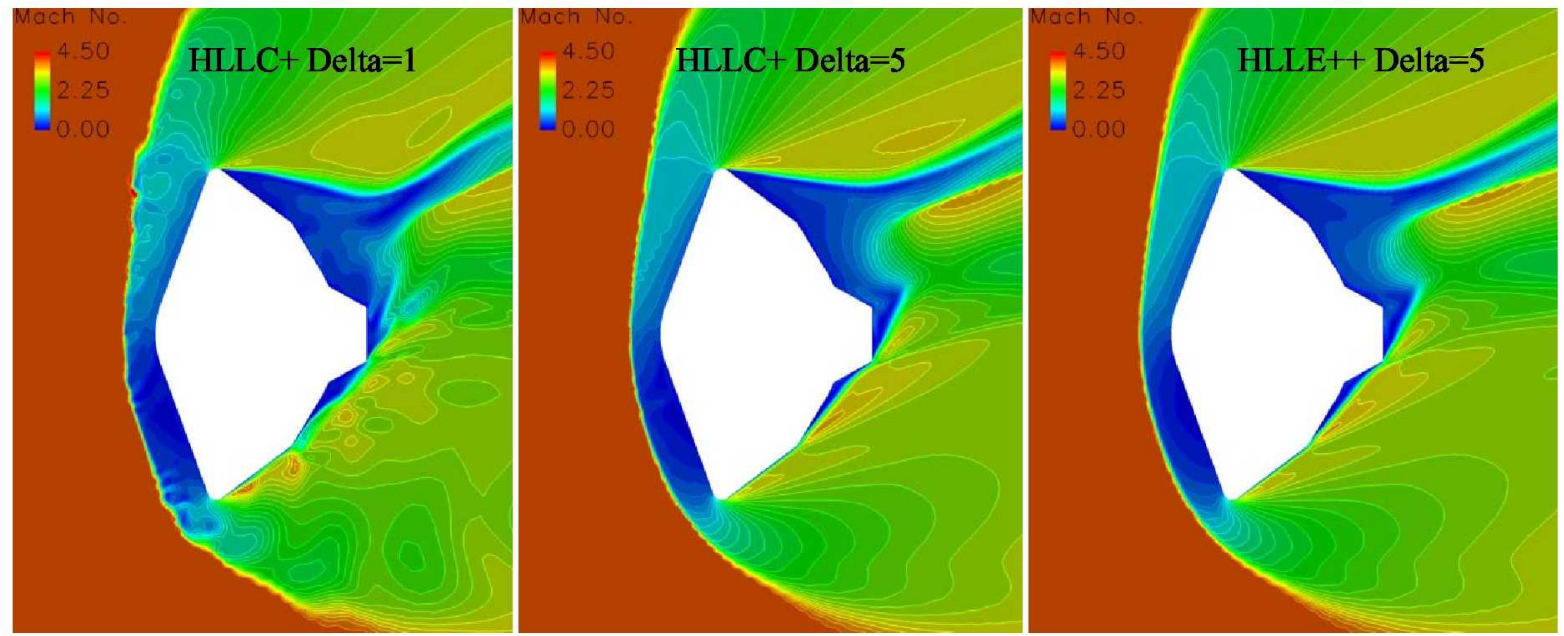

Figure 36. Mach number on the symmetry plane for the non-shock aligned grid.
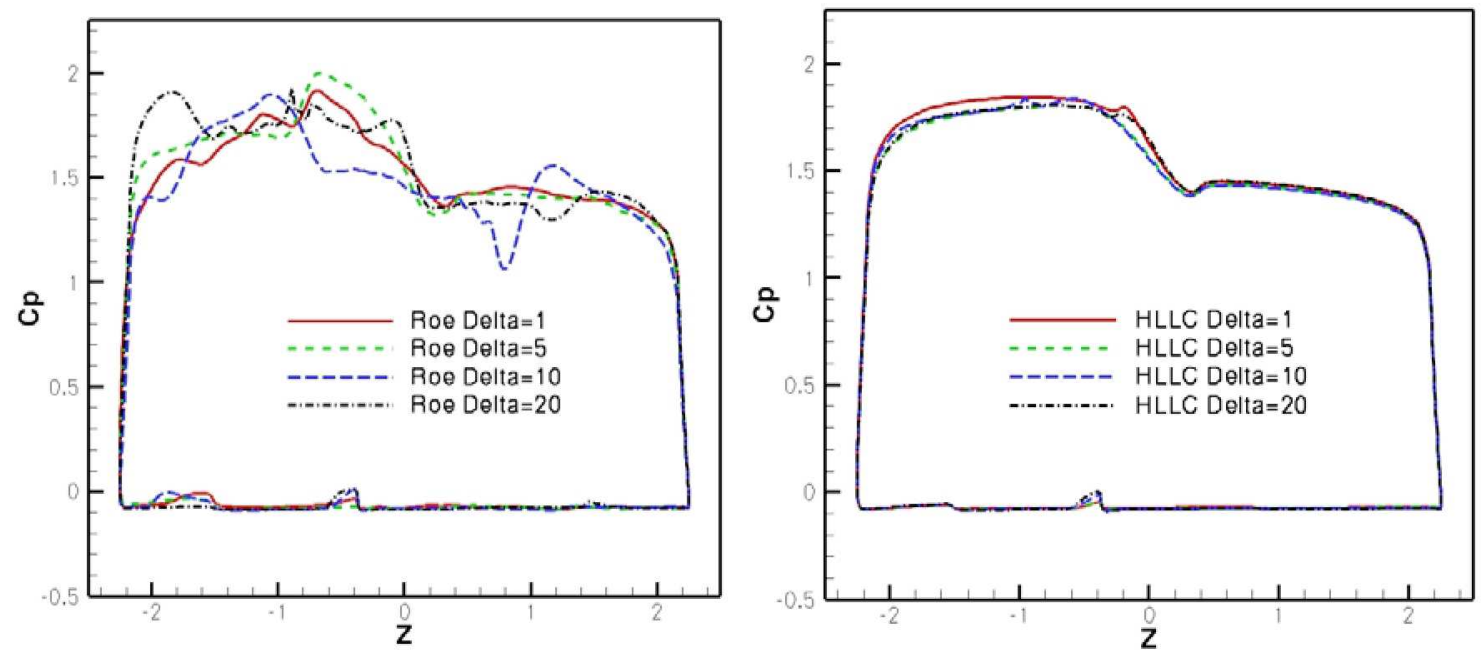

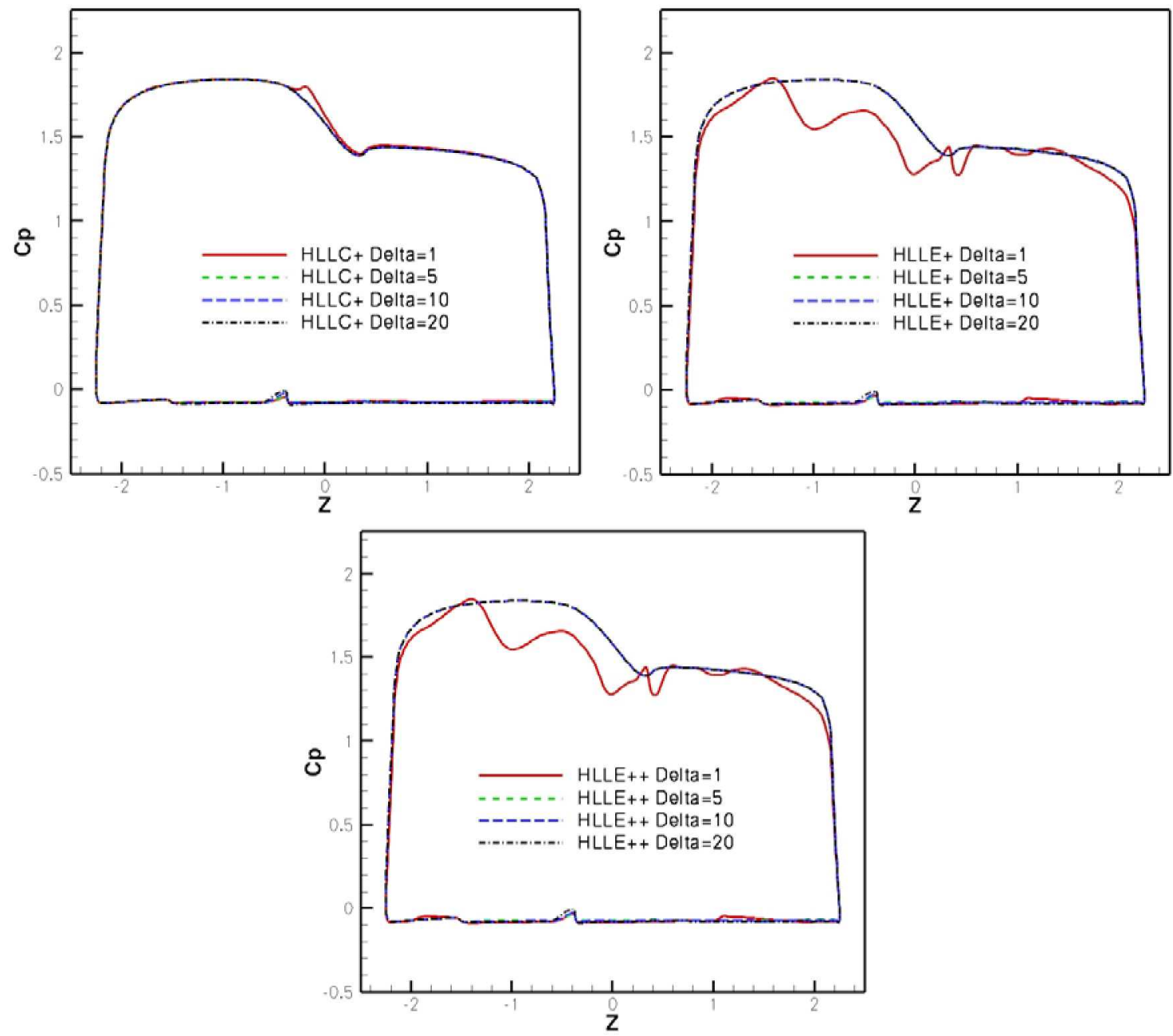

Figure 37. Pressure coefficient on the symmetry plane for the shock aligned grid.
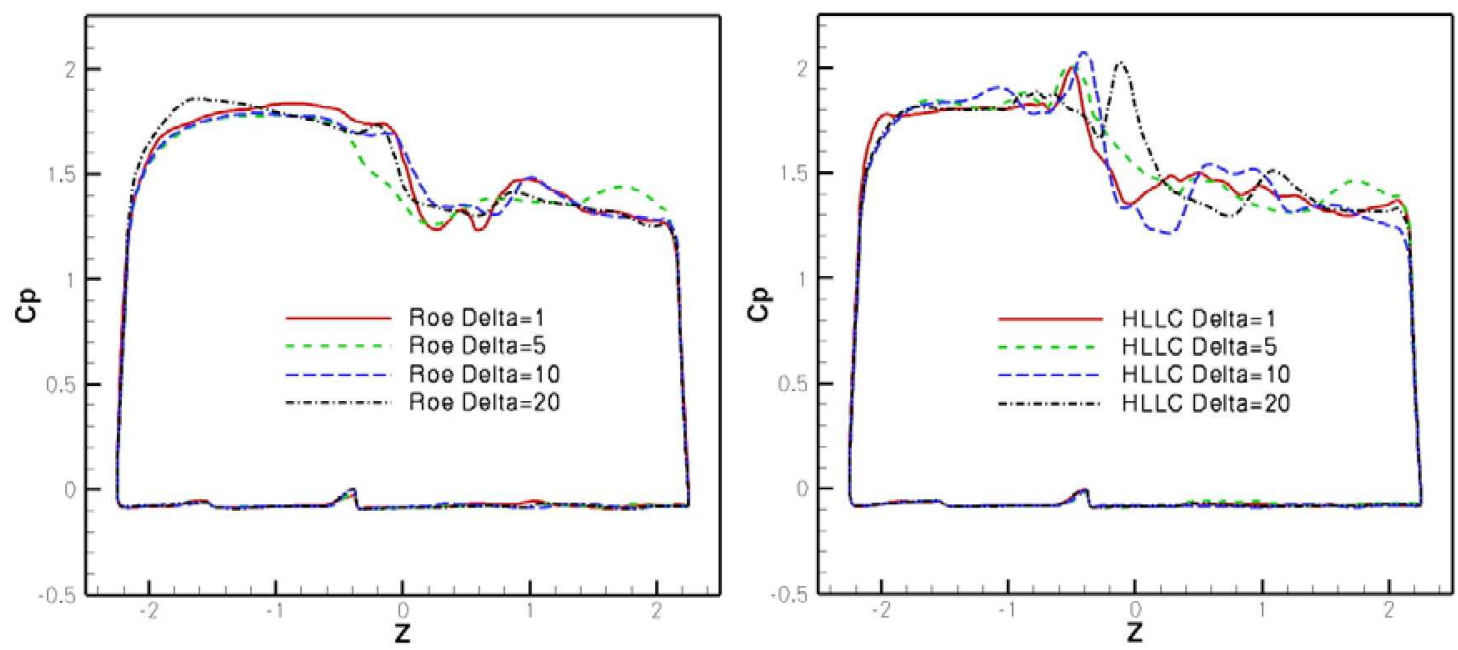

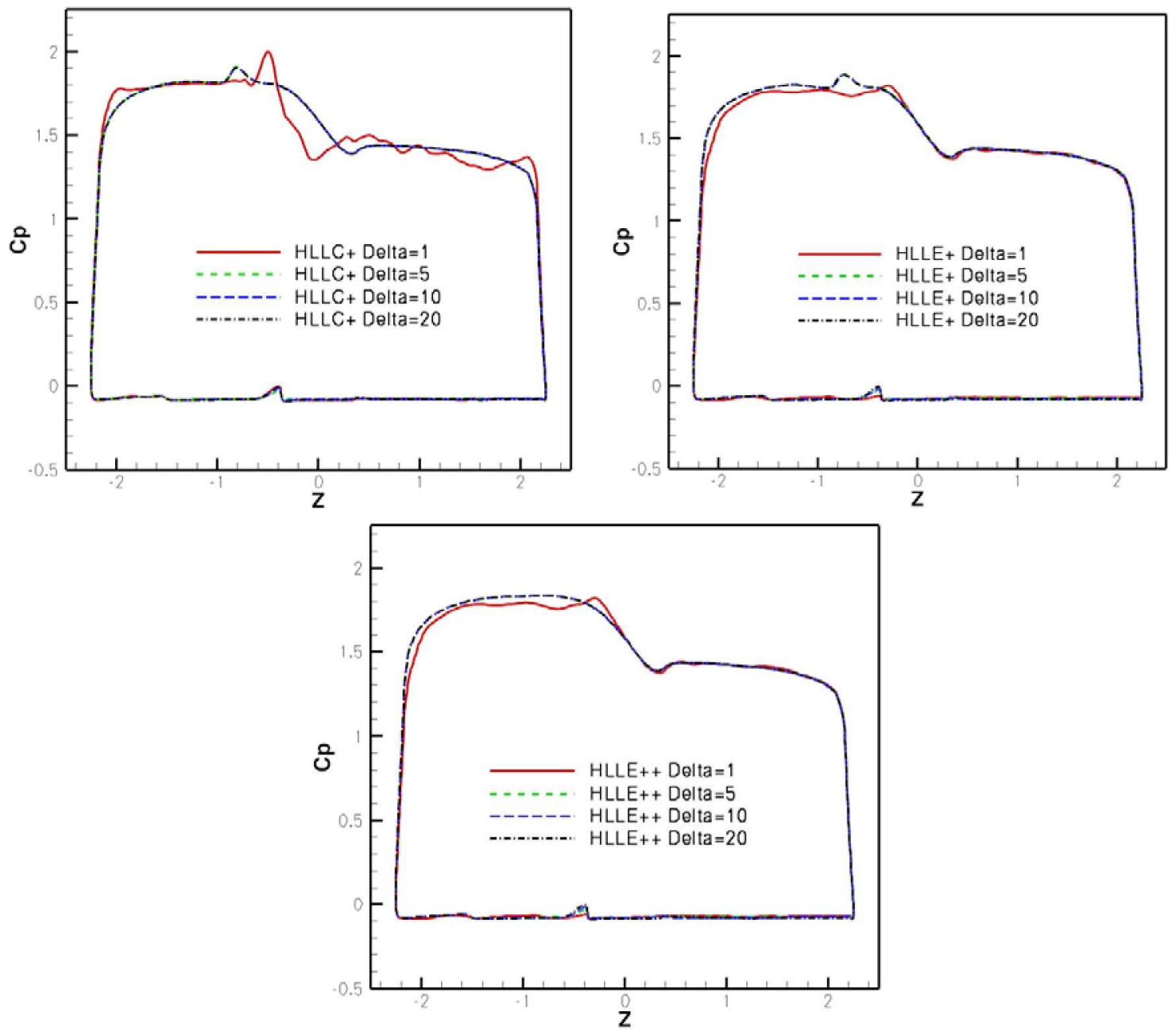

Figure 38. Pressure coefficient on the symmetry plane for the non-shock aligned grid.

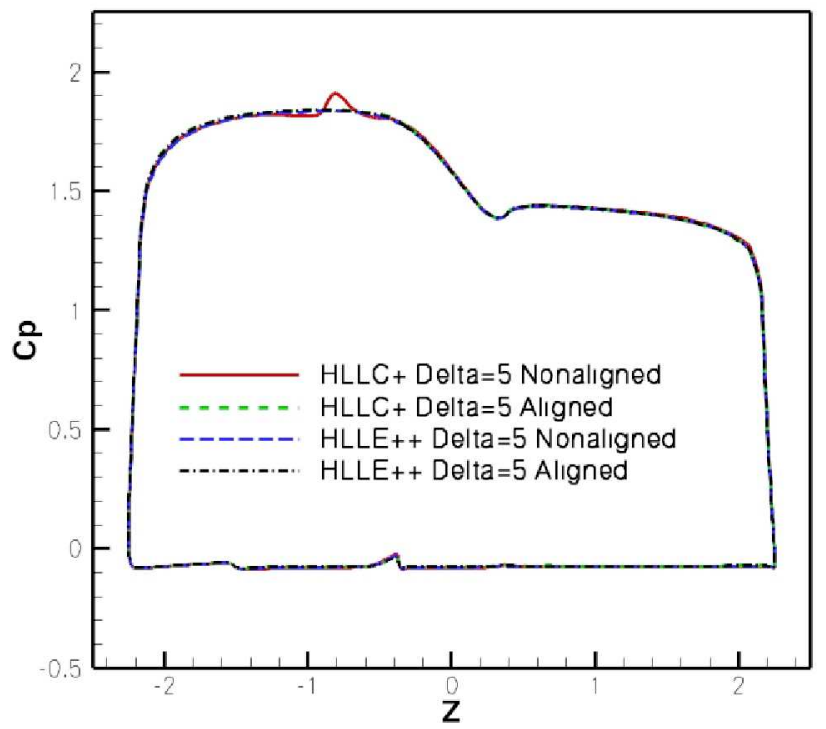

Figure 39. Pressure coefficient on the symmetry plane for both the shock aligned and non-shock aligned grids. 


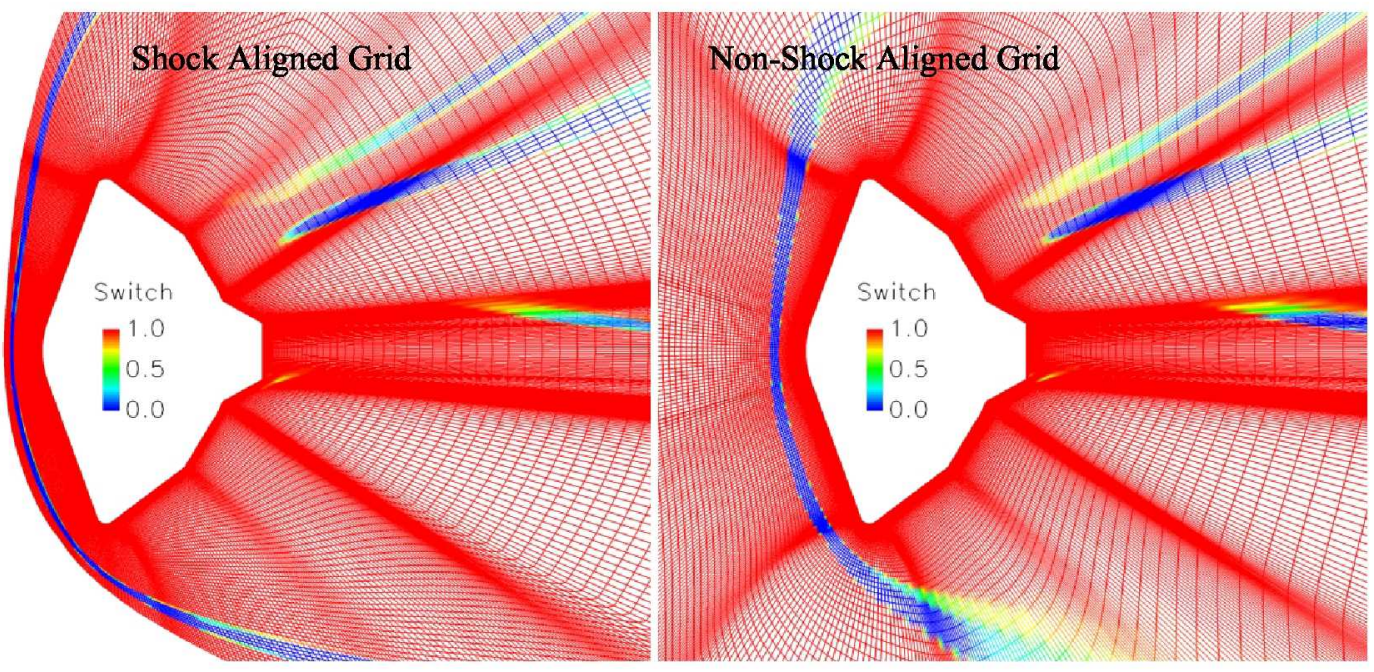

Figure 40. $\beta_{\text {New }}$ for the capsule for $D E L T A=5$. 